\title{
Stabilization of Austenitic Stainless Steel
}

\author{
By Samuel J. Rosenberg and John H. Darr
}

\begin{abstract}
A study was made of the resistance to intergranular attack of $2318 \% \mathrm{Cr}-10 \% \mathrm{Ni}$ austenitic corrosion-resisting steels in 12 different initial conditions. Susceptibility to intergranular attack was determined after seven sensitizing treatments; followed by exposure for a maximum of 14 days in a boiling acidified copper sulfate solution.

It was found that maximum susceptibility to intergranular attack was developed by sensitizing either 8 or 21 days at $1,020^{\circ} \mathrm{F}$. The straight carbon austenitic steels were quite vulnerable to attack, although decrease in carbon content decreased the degree of vulnerability. The columbium- and titanium-treated steels were satisfactorily resistant to attack provided the $\mathrm{Cb} / \mathrm{C}$ or $\mathrm{Ti} / \mathrm{C}$ ratios were sufficiently high. These ratios varied, depending on the initial condition of the steel. The carbon content of the treated steels had no influence upon the resistance to intergranular attack, the predominating factor being the $\mathrm{Cb} / \mathrm{C}$ or $\mathrm{Ti} / \mathrm{C}$ ratio.
\end{abstract}

\section{Introduction}

An undesirable characteristic of the austenitic stainless steels is their susceptibility to intergranular embrittlement after exposure to moderately elevated temperatures. This susceptibility to embrittlement may be decreased or eliminated, i. e., the steels may be stabilized against intergranular embrittlement, by the addition of titanium or columbium, usually in conjunction with a stabilizing heat treatment.

A diversity of opinion as to the relative amounts of titanium or columbium necessary for effective stabilization, the injurious effect of carbon content, and the necessity for stabilizing heat treatments, led the Bureau of Aeronautics, Navy Department, to request the National Bureau of Standards to undertake a study of factors affecting the stabilization of the $18-8$ type of steels. This paper summarizes the results of such an investigation.

\section{Theory of Sensitization and Stabiliza- tion}

Intergranular embrittlement (or corrosionthe terms are usually used synonomously) may be considered as a disease of 18-8 steels, although it is not peculiar to these steels alone. Specifi- cally, this type of corrosion is particularly pronounced in some 18-8 steels that have been subjected to moderately elevated temperatures $\left(700^{\circ}\right.$ to $1,400^{\circ} \mathrm{F}$ ) and are either simultaneously or subsequently subjected to corrosive conditions. Exposure to these elevated temperatures causes the precipitation of what are generally conceded to be chromium carbides at the grain boundaries, and steels having such carbide precipitation have been found to be more or less susceptible to intergranular corrosion, whereas steels that do not exhibit this structure are generally immune.

Annealed 18-8 stainless steel (quenched from temperatures in the neighborhood of $1,800^{\circ}$ to $\left.2,000^{\circ} \mathrm{F}\right)$ theoretically consists of metastable austenite, in this case a supersaturated solid solution of carbon or of chromium carbide in chromium-nickel austenite. Upon reheating to moderately elevated temperatures, chromium carbide $\left(\mathrm{Cr}_{4} \mathrm{C}\right)$ precipitates at the grain boundaries. One school of thought believes that this precipitation reduces the chromium content of the metal near the grain boundaries to a level below that necessary to resist corrosion. This appears to be the most prevalent view. Others believe that internal strains resulting from the precipitation of carbides, and from the formation of alpha iron from the metastable austenite, are the cause of intergranular corrosion. Still another belief is 
that the electrolytic effect resulting from a difference in potential between grains and grain boundaries is responsible. Regardless of the underlying theory, however, it is generally agreed that the presence of chromium carbides distributed in the form of a thin, nearly continuous network at the grain boundaries is an indication of material that is susceptible to intergranular corrosion. The larger isolated carbides that result after sufficient time at temperature has been allowed from coalescence of the precipitated carbides and for replenishment by diffusion of chromium in the depleted areas do not appear to be associated with intergranular corrosion.

The earliest efforts to prevent intergranular embrittlement were directed toward the manufacture of 18-8 with low carbon content on the theory that precipitation of chromium carbide would thereby be minimized or even eliminated $[1,2,3] .^{1} \quad$ It was also suggested that a treatment at about $1,600^{\circ} \mathrm{F}$ resulted in a decreased susceptibility to intergranular corrosion upon subsequent exposure at lower temperatures. This stabilizing (sometimes termed desensitizing) treatment was presumed to precipitate sufficient chromium carbides as coalesced particles, and also to permit diffusion of chromium, so that the amount of carbon that would be available to precipitate as fine chromium carbides at lower (sensitizing) temperatures would be negligible.

It was also reported that decreasing the austenitic grain size diminished the severity of intergranular attack [4] by providing extra grain boundary area for precipitation of carbides; that cold rolled material was more resistant to intergranular attack [3] because of the availability of numerous slip planes for the precipitation of carbides upon subsequent heating; and that the addition of elements that caused the formation of delta ferrite was also beneficial [5] in that, because of the lower solubility for carbides, precipitation occurred in the areas of delta ferrite.

The most commonly used method of preventing intergranular corrosion in 18-8 consists in adding a strongly carbide-forming element to the steel. The function of this element is to combine with the carbon, thus allowing the chromium to remain in solid solution in the austenite. To be effective this alloy carbide should be less soluble in the

\footnotetext{
1 Figures in brackets indicate the literature references at the end of this paper.
}

chromium-nickel austenite than is the chromium carbide. The two elements most frequently used are titanium and columbium $[6,7]$. It is known that these elements are more strongly carbide forming than is chromium, and evidence is available that the titanium and columbium carbides are considerably less soluble in $18-8$ austenite than is chromium carbide. Theoretically, if the carbon of 18-8 stainless steel is fixed by either of these stabilizing elements, that is, precipitated as $\mathrm{TiC}$ or $\mathrm{CbC}$, no chromium carbide would precipitate at the grain boundaries upon subsequent reheating to sensitizing temperatures, and the steel thereafter would exhibit no intercrystalline embrittlement upon exposure to a corroding medium.

The atomic weight of carbon is 12.01 , and that of titanium is 49.90 , and since titanium forms a carbide corresponding to $\mathrm{TiC}$, it is necessary, theoretically, to have about four times as much titanium as carbon in order to fix all the carbon as titanium carbide. Columbium has an atomic weight of 92.91, and as columbium forms a carbide corresponding to $\mathrm{CbC}$, the theoretical minimum amount of columbium that must be added to fix the carbon is slightly less than eight times the amount of carbon.

Stabilization consists in reheating the $\mathrm{Cb}-$ or Ti-treated steels within the temperature range $1,550^{\circ}$ to $1,800^{\circ} \mathrm{F}$, usually at about $1,600^{\circ} \mathrm{F}$. At these temperatures, the precipitation of titanium and columbium carbides within the austenitic grains is facilitated. The slight amount of carbon remaining in solid solution after this treatment is (theoretically) insufficient to cause any deleterious effects (in the form of $\mathrm{Cr}_{4} \mathrm{C}$ precipitated at the grain boundaries) upon subsequent reheating to sensitizing temperatures.

\section{Materials}

Most of the steels used in this investigation were experimental melts, although a few commercial steels were included. The experimental steels were melted in an induction furnace and poured into 3-in. square big end up tapered molds equipped with hot tops. Each experimental heat weighed about 75 pounds, and all were made in the foundry of the Naval Research Laboratory. The ingots were shaped on all four sides as much as necessary to produce clean surfaces, following which they were hot forged to slabs about 3 in. 
wide by $1 \mathrm{in.} \mathrm{thick.} \mathrm{Some} \mathrm{of} \mathrm{these} \mathrm{ingots} \mathrm{were}$ forged at the Rustless Iron and Steel Division, American Rolling Mill Co., Baltimore, Md.; all others were forged at the Naval Research Laboratory. The slabs were surface ground on four sides and rolled into 0.050-in. strip at the American Rolling Mill Co., Middletown, Ohio, the schedule of operations being as follows:

(1) Hot roll at $2,000^{\circ}$ to $2,200^{\circ} \mathrm{F}$ to $0.125 \mathrm{in}$. and straighten.

(2) Anneal 6 minutes at $1,950^{\circ}$ to $2,000^{\circ} \mathrm{F}$ and air cool.

(3) Pickle.

(a) $2 \frac{1}{2}$ minutes in 10 -percent $\mathrm{H}_{2} \mathrm{SO}_{4}$ at $190^{\circ} \mathrm{F}$.

(b) 45 minutes in caustic permanganate at $200^{\circ} \mathrm{F}$.

(c) 10 minutes in $1 \frac{1}{2}$-percent $\mathrm{HF}$ plus 10 percent $\mathrm{HNO}_{3}$ at $130^{\circ} \mathrm{F}$.

(4) Cold roll to $0.080 \mathrm{in}$. and straighten.

(5) Anneal 6 minutes at $1,950^{\circ}$ to $2,000^{\circ} \mathrm{F}$ and air cool.

(6) Pickle.

(b) and (c) only under (3) above.

(7) Cold roll to $0.050 \mathrm{in}$. and straighten.

The final strip thus had a cold reduction of $37 \frac{1 / 2}{12}$ percent. Although the sequence of fabrication was not known, all commercial steels were furnished in cold rolled strips $0.050 \mathrm{in}$. thick, the same as the experimental steels.

Chemical analyses of all the steels were made on samples cut from the finished strip; these analyses are given in table 1 . All experimental steels were made to the base analysis of 18 percent chromium, 10 percent nickel, 11/4 percent manganese, and 0.40 percent silicon.

During the progress of this investigation a question was raised as to the effect of nitrogen on the $\mathrm{Cb} / \mathrm{C}$ or $\mathrm{Ti} / \mathrm{C}$ ratio. Analyses for nitrogen were therefore made, both chemically and by vacuum fusion. It is known that nitrogen combines with both titanium and columbium. The acid-soluble and acid-insoluble nitrogen can be separated by treatment of the steel with dilute sulfuric acid. For purposes of calculation (as noted in table 1), it was assumed that all of the acid insoluble nitrogen was combined with either titanium or columbium, as the case might be. Considering the fact that the steels that contained no titanium or columbium also contained no acid insoluble nitrogen, this assumption appeared to be quite plausible. The revised percentages of titanium and columbium and the revised ratios of $\mathrm{Ti} / \mathrm{C}$ and $\mathrm{Cb} / \mathrm{C}$ were calculated after allowing for the amounts of titanium and columbium combined with the acid insoluble nitrogen and are included in table 1 .

\section{Procedure}

Specimens 3 in. long by $1 / 2$ in. wide and 0.050 in. nominal thickness were taken longitudinally to the direction of rolling. A small hole was punched in one end of each specimen so that groups of specimens could be strung on wire for sensitizing treatments. All such treatments were carried out in furnaces of the vertical muffle type, electrically heated, and constructed especially for this work. The inside dimensions of the muffles were $4 \mathrm{in}$. diameter by $18 \mathrm{in}$. long. Temperature variations within the working length of each furnace were less than $10^{\circ} \mathrm{F}$. Each furnace was controlled by an individual recording potentiometer controller.

Corrosion tests for development of intergranular embrittlement were conducted in a boiling acidified copper sulfate solution contained in 2-liter widemouthed Erlenmeyer flasks. These flasks were equipped with ground glass joints for fitting the reflux condensers. Specimens were laid in glass racks so that there was no metallic contact between specimens. No more than seven specimens were placed in an individual flask, and material of straight $18-10,18-10 \mathrm{Cb}$, and $18-10$ Ti was always segregated in different flasks. A minimum of $35 \mathrm{ml}$ of acidified copper sulfate solution per square inch of surface area was used. The solution was changed every 48 hours at which time all specimens were examined; those showing definite evidence of intergranular attack were removed. Where evidence of attack, as indicated by a change in color of the solution, occurred in less than 2 days, the run was interrupted to remove the failed specimen or specimens, and the solution was changed. Maximum time of exposure in the acidified copper sulfate solution was 14 days.

Preliminary tests indicated the necessity of carefully controlling certain factors. The original choice of concentration for the boiling copper sulfate-sulfuric acid solution was $13 \mathrm{~g}$ of 
TABLE 1. Analyses of the test steels

Steels designated by the prefix " $\mathrm{S}$ " are laboratory melts; those designated by the prefix "C" are commercial melts.

\begin{tabular}{|c|c|c|c|c|c|c|c|c|c|c|c|c|c|c|c|c|c|}
\hline \multirow[b]{2}{*}{ Steel No. } & \multicolumn{7}{|c|}{ Percentage of- } & \multicolumn{2}{|c|}{ Ratio } & \multirow{2}{*}{$\begin{array}{l}\text { Percent- } \\
\text { age of ni- } \\
\text { trogen } \\
\text { (vacuum } \\
\text { fusion) }\end{array}$} & \multicolumn{3}{|c|}{$\begin{array}{l}\text { Percentage of nitrogen } \\
\text { (chemical analysis) }\end{array}$} & \multicolumn{2}{|c|}{$\begin{array}{l}\text { Percentage } \\
\text { (revised) }^{a}\end{array}$} & \multicolumn{2}{|c|}{$\begin{array}{l}\text { Ratio (re- } \\
\text { vised) }{ }^{a}\end{array}$} \\
\hline & $\mathrm{C}$ & Mn & $\mathrm{Si}$ & $\mathrm{Cr}$ & $\mathrm{Ni}$ & $\mathrm{Cb}$ & $\mathrm{Ti}$ & $\mathrm{Cb} / \mathrm{C}$ & $\mathrm{Ti} / \mathrm{C}$ & & Total & $\begin{array}{l}\text { Acid } \\
\text { soluble }\end{array}$ & $\begin{array}{l}\text { Acid in- } \\
\text { soluble } \\
\text { (by dif- } \\
\text { ference) }\end{array}$ & $\mathrm{Cb}$ & $\mathrm{Ti}$ & $\mathrm{Cb} / \mathrm{C}$ & $\mathrm{Ti} / \mathrm{C}$ \\
\hline $\mathrm{C}-1 \ldots$ & 0.025 & 0.70 & 0.50 & 19.7 & 8.8 & & & & & 0.041 & 0.039 & 0.039 & 0.000 & & & & \\
\hline $\mathrm{C}-10 \ldots$ & .026 & .59 & .40 & 17.2 & 12. 6 & & & & & .026 & .026 & .025 & .001 & & & & \\
\hline S-37 $\ldots$ & .044 & 1. 10 & .33 & 17.8 & 10.5 & & & & & .049 & .053 & .053 & .000 & & & & \\
\hline S-4..- & .072 & 1. 13 & .45 & 18.0 & 10. 2 & & & & & .036 & .036 & .035 & .001 & & & & \\
\hline S $-23 \ldots$ & .113 & 1. 11 & .37 & 17.6 & 10.2 & & & & & .041 & .039 & .039 & .000 & & & & \\
\hline S $-26 \ldots$ & .068 & 1. 21 & .37 & 17.8 & 10.0 & 0.50 & & 7.4 & & .065 & .061 & .041 & .020 & 0.37 & & 5.4 & \\
\hline S-6 $\ldots$ & .070 & 1. 23 & .54 & 17.6 & 10.1 & .61 & & 8.7 & & .056 & .054 & .034 & .020 & .48 & & 6.9 & \\
\hline S-12 $\ldots$ & .074 & 1. 23 & .38 & 17.9 & 10.0 & .75 & & 10.1 & & .051 & .047 & .024 & .023 & .60 & & 8.1 & \\
\hline $\mathrm{C}-3 \ldots$ & .060 & 1. 69 & 67 & 17.7 & 11.0 & .71 & & 11.8 & & .032 & .035 & .015 & .020 & .58 & & 9.7 & \\
\hline S-27 & .087 & 1. 19 & .40 & 17.8 & 10.1 & .76 & & 8.7 & & .059 & .057 & .030 & .027 & .58 & & 6.7 & \\
\hline S-28 ... & .115 & 1. 22 & .40 & 17.7 & 9.9 & .92 & & 8.0 & & .045 & .045 & .022 & .023 & .77 & & 6. 7 & \\
\hline $\mathrm{S}-17 \ldots \ldots$ & .117 & 1. 21 & .40 & 18.0 & 10.1 & 1.14 & & 9.7 & & .039 & .040 & .016 & .024 & .98 & & 8.3 & \\
\hline S-18 $\ldots \ldots$ & .132 & 1. 18 & .40 & 17.9 & 10.1 & 1.47 & & 11.1 & & .044 & .046 & .014 & .032 & 1. 26 & & 9.5 & - \\
\hline S-34 & .067 & 1. 17 & .45 & 17. 7 & 10.2 & & 0.26 & & 3.9 & .031 & .039 & .001 & .038 & & 0.13 & & 1. 9 \\
\hline S-21 ... & .071 & 1. 49 & .41 & 17.9 & 10.1 & & .32 & & 4.5 & .027 & .031 & .002 & .029 & & .22 & & 3.1 \\
\hline $\mathrm{C}-6 \ldots$ & .064 & 1. 32 & .40 & 18.3 & 10.0 & & .35 & & 5.5 & .012 & .013 & .002 & .011 & & .31 & & 4. 8 \\
\hline S-39 $\ldots$ & .065 & 1. 23 & .53 & 18. 2 & 10.1 & & .36 & & 5.5 & .023 & .023 & .001 & .022 & & .28 & & 4.3 \\
\hline $\mathrm{C}-2$ & .070 & 1. 52 & .50 & 17.8 & 9.6 & & .50 & & 7.1 & .010 & .010 & .001 & .009 & & .47 & & 6. 7 \\
\hline C $-8 \ldots$ & .075 & 1. 25 & .52 & 18.0 & 10.6 & & .59 & & 7.9 & .006 & .007 & .001 & .006 & & .57 & & 7. 6 \\
\hline $\mathrm{S}-25 \ldots$ & .082 & 1. 23 & .40 & 17.7 & 9.9 & & .37 & & 4.5 & .017 & .022 & .001 & .021 & & .30 & & 3.7 \\
\hline S-35 $\ldots$ & .107 & 1. 29 & .42 & 17.5 & 9.7 & & .44 & & 4. 1 & .012 & .016 & .001 & .015 & & .39 & & 3. 6 \\
\hline S-36 .... & .109 & 1.35 & .48 & 17.5 & 10.0 & & .54 & & 5.0 & .022 & .018 & .001 & .017 & & .48 & & 4. 4 \\
\hline S-32 ....... & .105 & 1.44 & .40 & 18.0 & 9.9 & & .61 & & 5.8 & .016 & .017 & .001 & .016 & & .56 & & 5. 3 \\
\hline
\end{tabular}

a The revised percentages of Ti and $\mathrm{Cb}$ were calculated by assuming that all of the acid insoluble nitrogen was combined as TiN or CbN. According to the atomic weights of these elements, 1 part of nitrogen will combine with 3.43 parts of titanium or 6.64 parts of columbium by weight. Multiplying these figures by the amount of acid insoluble nitrogen in the titanium-and eolumbium-treated steels, respectively, and subtracting the results from the amounts of titanium and columbium present in the steels gives the revised amounts of titanium or columbium available for combination with earbon. The revised $\mathrm{Ti} / \mathrm{C}$ and $\mathrm{Cb} / \mathrm{C}$ ratios were then obtained by dividing the revised percentages of titanium and columbium by the carbon contents

$\mathrm{CuSO}_{4} \cdot 5 \mathrm{H}_{2} \mathrm{O}, 47 \mathrm{ml}$ of concentrated $\mathrm{H}_{2} \mathrm{SO}_{4}$, and distilled water to make 1 liter of solution. This solution, which has been used by some investigators, corresponded to 0.8 percent of $\mathrm{CuSO}_{4}$ and 8.2 percent of $\mathrm{H}_{2} \mathrm{SO}_{4}$, and the ratio of $\mathrm{CuSO}_{4}$ to $\mathrm{H}_{2} \mathrm{SO}_{4}$ proved too low to prevent surface corrosion in many of the steels. Since the resultant corrosion complicated the evaluation of intergranular embrittlement, this solution was discarded in favor of that containing $100 \mathrm{~g}$ of $\mathrm{CuSO}_{4} \cdot 5 \mathrm{H}_{2} \mathrm{O}, 100 \mathrm{ml}$ of concentrated $\mathrm{H}_{2} \mathrm{SO}_{4}$ (sp gr 1.84 ), and $900 \mathrm{ml}$ of distilled water. This solution corresponded to 5.4 percent of $\mathrm{CuSO}_{4}$ and 15.4 percent of $\mathrm{H}_{2} \mathrm{SO}_{4}$ by weight.

The scale that formed on specimens during sensitization was influenced by both time and temperature. In order to eliminate the variable which scale would introduce, it was decided to remove all scale. Polishing was tried and proved to be too time-consuming; pickling was therefore adopted with a schedule of operations as follows: (1) Pickle in 8 percent $\mathrm{H}_{2} \mathrm{SO}_{4}+4$ percent $\mathrm{HCl}$ at $160^{\circ} \mathrm{F}$ for 10 minutes; wash, scrub, and dry; (2) pickle in 10 percent $\mathrm{HNO}_{3}+1$ percent $\mathrm{HF}$ at $160^{\circ} \mathrm{F}$ for 5 minutes; wash, scrub, and dry; (3) passivate in 20 percent $\mathrm{HNO}_{3}$ at $125^{\circ} \mathrm{F}$ for 15 minutes; wash and dry.

To ascertain whether the method of scale removal influenced the susceptibility to intergranular attack, check tests were made on certain specimens both as pickled and as polished. These tests showed that the method of scale removal 
had no detectable effect on the specimens that were extremely vulnerable to intergranular embrittlement. With specimens that were moderately vulnerable, it appeared that the extent of intergranular attack was greater in specimens that had been pickled than in specimens that has been polished. Typical microstructures of such specimens after corrosion are shown in figure 1 . Since it is frequently commercial practice to finish stainless steels by pickling, it appeared logical to test the steels as finished in that manner.

The susceptibility to intergranular attack of the various steels was determined for 12 different initial conditions, as listed in tables 2 to 24 . A few steels were tested in only five different initial conditions, as may be seen from the particular table involved.

In the first series of tests (carried out on the cold rolled steels), specimens from each steel were tested in 16 sensitized conditions. Four sensitizing temperatures were used $-840^{\circ}, 1,020^{\circ}, 1,200^{\circ}$, and $1,380^{\circ} \mathrm{F}$. Single specimens were held at each sensitizing temperature for 2 hours, 2,8 , and 21 days. After exposure to the boiling copper sulfate solution, it was apparent that certain of these sensitizing treatments were quite innocuous and could be discarded. It was decided, therefore, to decrease the number of sensitizing conditions to seven in all-these were 21 days at $840^{\circ} \mathrm{F}$; 2 hours, 2,8 , and 21 days at $1,020^{\circ} \mathrm{F}$; and 2 hours and 2 days at $1,200^{\circ} \mathrm{F}$. A control specimen, not sensitized, was included in each series.

Evaluation of intergranular embrittlement was made by observations of: (a) change in electrical resistivity, (b) loss in metallic ring when the test specimen was dropped on a steel plate, (c) extent of cracks occurring after bend tests of $180^{\circ}$, and (d) appearance of the corroded sections under the microscope. The interpretation of these various tests was frequently influenced by the personal factor, particularly in cases where only slight evidence of failure existed. Frequently, also, evidence of failure by one method was not corroborated by another.
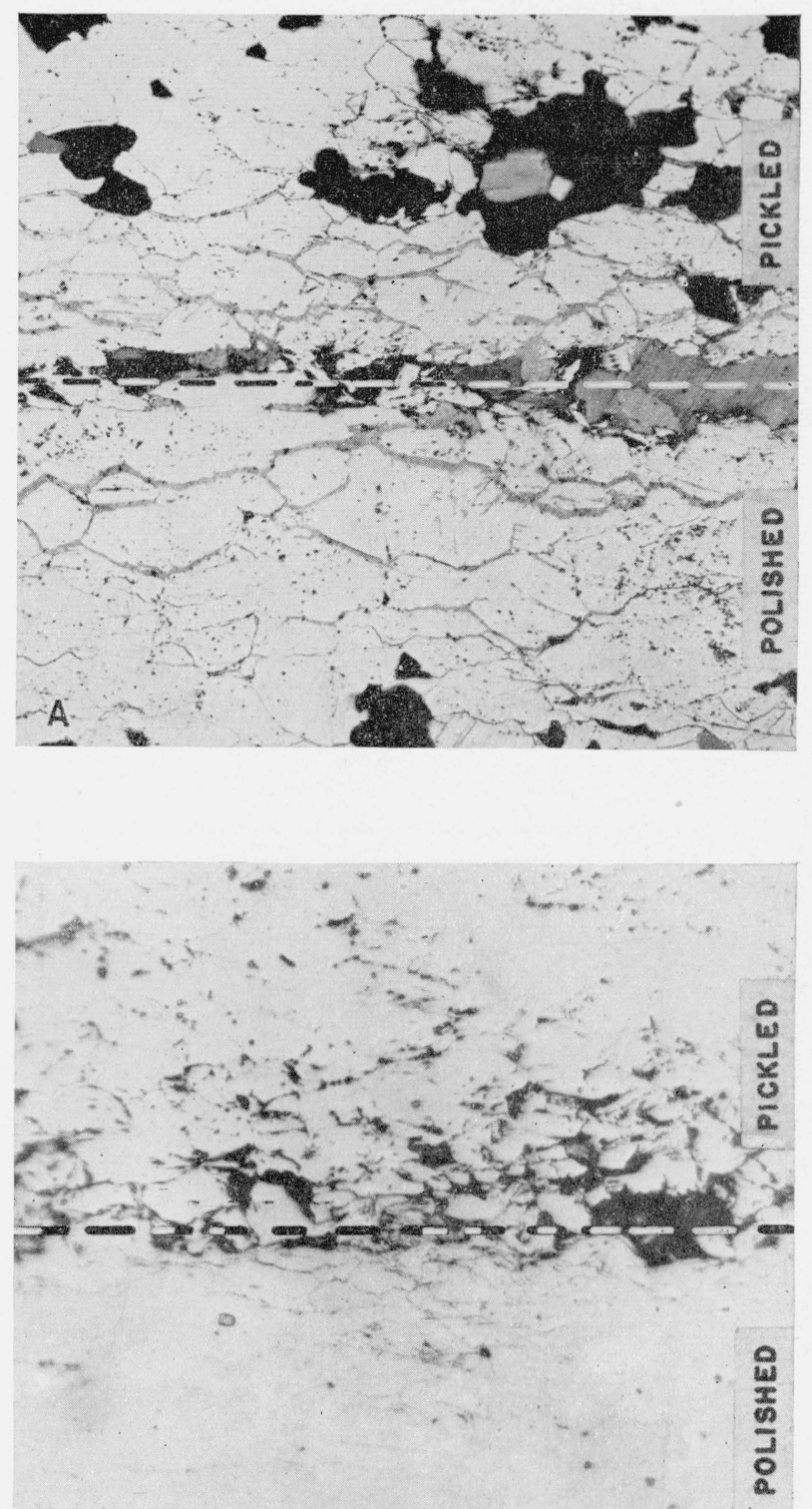

B

FIGURE 1. Effect of method of scale removal on susceptibility to intergranular attack.

$A$, Extremely vulnerable material after 2 days in the boiling acidified copper sulfate solution. Unetched. $\times 100$. $B$, Less vulnerable material after 6 days in the boiling acidified copper sulfate solution. Unetched. $\times 500$. 
TABLE 2. Effects of various treatments upon the susceptibility to intergranular attack of steel $C-1$

(Carbon $=0.025 \%$-no stabilizing clement)

\begin{tabular}{|c|c|c|c|c|c|c|c|c|c|}
\hline \multicolumn{2}{|c|}{ Treatment of steel subsequent to cold-rolling } & \multicolumn{8}{|c|}{ Sensitizing treatment } \\
\hline Annealing & Stabilizing & None & $\begin{array}{l}21 \text { days } \\
\text { at } 840^{\circ} \mathrm{F}\end{array}$ & $\begin{array}{l}2 \text { hours } \\
\text { at } 1,020^{\circ} \mathrm{F}\end{array}$ & $\begin{array}{l}2 \text { days } \\
\text { at } 1,020^{\circ} \mathrm{F}\end{array}$ & $\begin{array}{c}8 \text { days } \\
\text { at } 1,020^{\circ} \mathrm{F}\end{array}$ & $\begin{array}{c}21 \text { days } \\
\text { at } 1,020^{\circ} \mathrm{F}\end{array}$ & $\begin{array}{c}2 \text { hours } \\
\text { at } 1,200^{\circ} \mathrm{F}\end{array}$ & $\begin{array}{c}2 \text { days } \\
\text { at } 1,200^{\circ} \mathrm{F}\end{array}$ \\
\hline None..... & None & A & $\mathrm{A}$ & A & $\mathrm{D}-$ & $\mathbf{E}+$ & $\mathrm{D}-$ & A & A \\
\hline Do... & $1 / 2$ hour at $1,600^{\circ} \mathrm{F}, \mathrm{A} . \mathrm{C}$ & A & $A-$ & A & $\mathrm{C}$ & $\mathrm{D}-$ & $\mathrm{D}-$ & $\mathrm{A}$ & A \\
\hline $1 / 2$ hour at $1,800^{\circ} \mathrm{F}, \mathrm{W} \cdot \mathrm{Q}$ & None & A & A & A & $\mathrm{DE}$ & DE & $\mathrm{E}$ & $A-$ & A \\
\hline Do & $1 / 2$ hour at $1,600^{\circ} \mathrm{F}, \mathrm{A} . \mathrm{C} \ldots$ & A & A & A & $\mathrm{B}$ & DE & $\mathrm{E}+$ & A & $\mathrm{A}$ \\
\hline Do......... & 2 hours at $1,600^{\circ} \mathrm{F}, \mathrm{A} . \mathrm{C}$ & A & $A-$ & A & $\mathrm{B}$ & DE & $\mathrm{DE}$ & $A-$ & A \\
\hline $1 / 2$ hour at $1,800^{\circ} \mathrm{F}, \mathrm{A}$. C & None & A & A & $A$ & $\mathrm{C}-$ & $\mathrm{D}$ & $\mathrm{CD}$ & $A-$ & A \\
\hline Do... & 1 hour at $1,600^{\circ} \mathrm{F}, \mathrm{A} . \mathrm{C}$ & A & A & $A$ & $\mathrm{C}-$ & $\mathrm{D}$ & $\mathrm{D}$ & A & $A-$ \\
\hline $3 \mathrm{~min}$, at $1,975^{\circ} \mathrm{F}, \mathrm{W}, \mathrm{Q}$ & None & A & A & A & $\mathrm{CD}$ & $\mathrm{D}$ & $\mathrm{CD}$ & A & A \\
\hline Do. & 1 hour at $1,600^{\circ} \mathrm{F}, \mathrm{A} . \mathrm{C} \ldots$ & A & A & A & $\mathrm{BC}$ & $\mathrm{CD}$ & $\mathrm{CD}$ & A & A \\
\hline $3 \mathrm{~min}$ at $1,975^{\circ} \mathrm{F}, \mathrm{A} . \mathrm{C}$ & None & A & A & A & $\mathrm{B}-$ & $\mathrm{DE}$ & $\mathrm{C}$ & A & A \\
\hline Do & $1 / 2$ hour at $1,600^{\circ} \mathrm{F}, \mathrm{A} . \mathrm{C} \ldots$ & A & A & A & $\mathrm{D}+$ & $\mathrm{D}$ & $\mathrm{D}$ & A & $A-$ \\
\hline Do & 2 hours at $1,600^{\circ} \mathrm{F}, \mathrm{A} . \mathrm{C} \ldots$ & A & A & A & $\mathrm{O}-$ & $\mathrm{D}-$ & $\mathrm{D}+$ & $A-$ & $A-$ \\
\hline
\end{tabular}

TABLE 3. Effect of various treatments upon the susceptibility to intergranular attack of steel C-10

(Carbon $=0.026 \%$-no stabilizing element)

\begin{tabular}{|c|c|c|c|c|c|c|c|c|c|}
\hline \multicolumn{2}{|c|}{ Treatment of steel subsequent to cold-rolling } & \multicolumn{8}{|c|}{ Sensitizing treatment } \\
\hline Annealing & Stabilizing & None & $\begin{array}{l}21 \text { days } \\
\text { at } 840^{\circ} \mathrm{F}\end{array}$ & $\begin{array}{c}2 \text { hours } \\
\text { at } 1,029^{\circ} \mathrm{F}\end{array}$ & $\begin{array}{c}2 \text { days } \\
\text { at } 1,020^{\circ} \mathrm{F}\end{array}$ & $\begin{array}{c}8 \text { days } \\
\text { at } 1,020^{\circ} \mathrm{F}\end{array}$ & $\begin{array}{c}21 \text { days } \\
\text { at } 1,020^{\circ} \mathrm{F}\end{array}$ & $\begin{array}{l}2 \text { hours } \\
\text { at } 1,200^{\circ} \mathrm{F}\end{array}$ & $\begin{array}{l}2 \text { days } \\
\text { at } 1,200^{\circ} \mathrm{F}\end{array}$ \\
\hline None.. & None & A & $\mathrm{B}+$ & A & $\mathrm{B}-$ & A & $A-$ & $A-$ & A \\
\hline Do & $1 / 2$ hour at $1,600^{\circ} \mathrm{F}, \mathrm{A} . \mathrm{C} \ldots$ & A & A & $A-$ & DE & $\mathrm{E}$ & $\mathrm{E}$ & $A-$ & $\mathrm{B}-$ \\
\hline $1 / 2$ hour at $1,800^{\circ} \mathrm{F}, \mathrm{W} . \mathrm{G}$ & None & $A-$ & A & A & $\mathrm{E}+$ & $\mathrm{E}$ & $\mathrm{E}$ & $\mathrm{AB}$ & $\mathrm{C}-$ \\
\hline Do & $1 / 2$ hour at $1,600^{\circ} \mathrm{F}, \mathrm{A} . \mathrm{C} \ldots$ & A & A & A & $\mathrm{E}+$ & $\mathrm{E}$ & $\mathrm{E}$ & A & $\mathrm{B}-$ \\
\hline Do & 2 hours at $1,600^{\circ} \mathrm{F}, \mathrm{A} . \mathrm{C} \ldots$ & $A-$ & A & A & $\mathrm{E}+$ & $\mathrm{E}$ & $\mathrm{E}$ & $A-$ & $\mathrm{BC}$ \\
\hline $1 / 2$ hour at $1,800^{\circ} \mathrm{F}, \mathrm{A}$. C & None & A & A & A & $\mathrm{E}+$ & $\mathrm{E}$ & $\mathrm{E}$ & $\mathrm{AB}$ & $\mathrm{CD}$ \\
\hline Do & 1 hour at $1,600^{\circ} \mathrm{F}, \mathrm{A} . \mathrm{C} \ldots$ & A & A & A & $\mathrm{E}$ & $\mathrm{E}$ & $\mathrm{E}$ & $A B$ & $\mathrm{C}$ \\
\hline $3 \mathrm{~min}$ at $1,975^{\circ} \mathrm{F}, \mathrm{W} . \mathrm{Q}$ - & None & A & A & A & $\mathrm{E}$ & $\mathrm{E}$ & $\mathrm{E}$ & $A-$ & $A-$ \\
\hline Do & 1 hour at $1,600^{\circ} \mathrm{F}, \mathrm{A} . \mathrm{C} \ldots$ & A & A & A & $\mathrm{E}+$ & $\mathrm{E}$ & $\mathrm{E}$ & $A-$ & $\mathrm{BC}$ \\
\hline $3 \mathrm{~min}$ at $1,975^{\circ} \mathrm{F}, \mathrm{A} . \mathrm{C}$ & None & A & A & A & $\mathrm{D}+$ & $\mathrm{E}$ & $\mathrm{E}$ & A & $\mathrm{BC}$ \\
\hline Do & $1 / 2$ hour at $1,600^{\circ} \mathrm{F}, \mathrm{A} . \mathrm{C} \ldots \ldots$ & A & A & A & $\mathrm{D}+$ & $\mathrm{E}$ & $\mathrm{E}$ & A & $\mathrm{C}+$ \\
\hline Do & 2 hours at $1,600^{\circ} \mathrm{F}, \mathrm{A} . \mathrm{C} \ldots \ldots$ & A & A & A & $\mathrm{D}$ & $\mathrm{E}$ & $\mathrm{E}$ & A & $\mathrm{C}-$ \\
\hline
\end{tabular}

TABLE 4. Effect of various treatments upon the susceptibility to intergranular attack of steel S-37

(Carbon $=0.044 \%$-no stabilizing element)

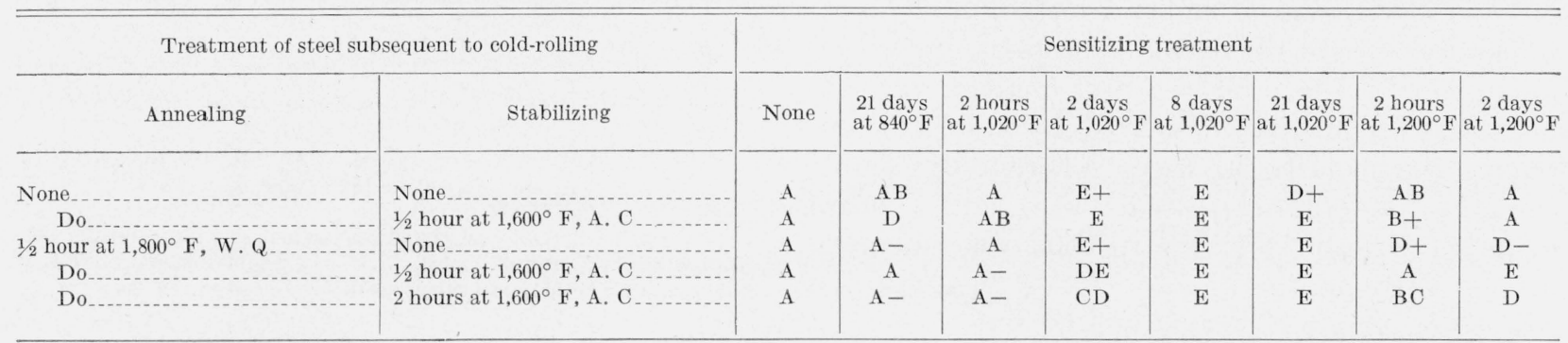


TABLE 5. Effect of various treatments upon the susceptibility to intergranular attack of steel S-4

(Carbon $=0.072 \%$-no stabilizing element)

\begin{tabular}{|c|c|c|c|c|c|c|c|c|c|}
\hline \multicolumn{2}{|c|}{ Treatment of steel subsequent to cold-rolling } & \multicolumn{8}{|c|}{ Sensitizing treatment } \\
\hline Annealing & Stabilizing & None & $\begin{array}{l}21 \text { days } \\
\text { at } 840^{\circ} \mathrm{F}\end{array}$ & $\begin{array}{l}2 \text { hours } \\
\text { at } 1,020^{\circ} \mathrm{F}\end{array}$ & $\begin{array}{c}2 \text { days } \\
\text { at } 1,020^{\circ} \mathrm{F}\end{array}$ & $\begin{array}{c}8 \text { days } \\
\text { at } 1,020^{\circ} \mathrm{F}\end{array}$ & $\begin{array}{c}21 \text { days } \\
\text { at } 1,020^{\circ} \mathrm{F}\end{array}$ & $\begin{array}{c}2 \text { hours } \\
\text { at } 1,200^{\circ} \mathrm{F}\end{array}$ & $\begin{array}{c}2 \text { days } \\
\text { at } 1,200^{\circ} \mathrm{F}\end{array}$ \\
\hline None.... & None & A & $\mathrm{D}+$ & $\mathrm{DE}$ & $\mathrm{E}$ & $\mathrm{E}$ & $\mathrm{E}$ & $\mathrm{E}$ & A \\
\hline Do & $1 / 2$ hour at $1,600^{\circ} \mathrm{F}, \mathrm{A} . \mathrm{C}$ & $\mathrm{B}$ & $\mathrm{E}$ & $\mathrm{E}$ & $\mathrm{E}$ & $\mathrm{E}$ & $\mathrm{E}$ & $\mathrm{CD}$ & A \\
\hline $1 / 2$ hour at $1,800^{\circ} \mathrm{F}, \mathrm{W} . \mathrm{Q} \ldots \ldots$ & None & A & $A-$ & A & $\mathrm{E}$ & $\mathrm{E}$ & $\mathrm{E}$ & $\mathrm{E}$ & $\mathrm{E}$ \\
\hline Do & $1 / 2$ hour at $1,600^{\circ} \mathrm{F}, \mathrm{A} . \mathrm{C}$ & $\mathrm{DE}$ & $\mathrm{E}$ & $\mathrm{E}$ & $\mathrm{E}$ & $\mathrm{E}$ & $\mathrm{E}$ & $\mathrm{E}$ & $\mathrm{E}$ \\
\hline Do & 2 hours at $1,600^{\circ}$ F. A. C & D & $\mathrm{E}$ & $\mathrm{E}$ & $\mathrm{E}$ & $\mathrm{E}$ & $\mathrm{E}$ & $\mathrm{E}$ & $\mathrm{E}+$ \\
\hline $1 / 2$ hour at $1,800^{\circ} \mathrm{F}, \mathrm{A} . \mathrm{C} \ldots$ & None & A & $\mathrm{D}$ & A & $\mathrm{E}$ & $\mathrm{E}$ & $\mathrm{E}$ & $\mathrm{E}$ & $\mathrm{E}$ \\
\hline Do & 1 hour at $1,600^{\circ} \mathrm{F}, \mathrm{A} . \mathrm{C}$ & $\mathrm{C}-$ & $\mathrm{F}$. & $\mathrm{E}$ & $\mathrm{E}$ & $\mathrm{E}$ & $\mathrm{E}$ & $\mathrm{E}$ & $\mathrm{D}$ \\
\hline $3 \mathrm{~min}$ at $1,975^{\circ} \mathrm{F}, \mathrm{W}, \mathrm{Q}$ & None & A & A & $\mathrm{A}$ & $\mathrm{DE}$ & $\mathrm{E}$ & $\mathrm{E}$ & $\mathrm{E}$ & $\mathrm{E}$ \\
\hline Do & 1 hour at $1,600^{\circ} \mathrm{F}, \mathrm{A}, \mathrm{C}$ & $\mathrm{D}$ & $\mathrm{E}$ & $\mathrm{E}+$ & $\mathrm{E}$ & $\mathrm{E}+$ & $\mathrm{E}+$ & $\mathrm{DE}$ & $\mathrm{E}$ \\
\hline $3 \mathrm{~min}$ at $1,975^{\circ} \mathrm{F}, \mathrm{A} . \mathrm{C}$ & None & A & A & A & $\mathrm{D}$ & $\mathrm{E}$ & E & $\mathrm{E}$ & $\mathrm{E}$ \\
\hline Do & $1 / 2$ hour at $1,600^{\circ} \mathrm{F}, \mathrm{A}, \mathrm{C}$ & $\mathrm{C}$ & $\mathrm{D}$ & D & $\mathrm{E}$ & $\mathrm{E}$ & $\mathrm{E}$ & $\mathrm{E}$ & $\mathrm{E}$ \\
\hline Do & 2 hours at $1,600^{\circ} \mathrm{F}, \mathrm{A} . \mathrm{C} \ldots \ldots$ & $\mathrm{C}+$ & $\mathrm{E}+$ & $\mathrm{E}+$ & $\mathrm{E}+$ & $\mathrm{E}$ & $\mathrm{E}$ & $\mathrm{E}$ & $\mathrm{E}$ \\
\hline
\end{tabular}

TABLE 6. Effect of various treatments upon the susceptibility to intergranular attack of steel S-23

(Carbon $=0.113 \%$-no stabilizing element)

\begin{tabular}{|c|c|c|c|c|c|c|c|c|c|}
\hline \multicolumn{2}{|c|}{ Treatment of steel subsequent to cold-rolling } & \multicolumn{8}{|c|}{ Sensitizing treatment } \\
\hline Annealing & Stabilizing & None & $\begin{array}{l}21 \text { days } \\
\text { at } 840^{\circ} \mathrm{F}\end{array}$ & $\begin{array}{c}2 \text { hours } \\
\text { at } 1,020^{\circ} \mathrm{F}\end{array}$ & $\begin{array}{c}2 \text { days } \\
\text { at } 1,020^{\circ} \mathrm{F}\end{array}$ & $\begin{array}{c}8 \text { days } \\
\text { at } 1,020^{\circ} \mathrm{F}\end{array}$ & $\begin{array}{c}21 \text { days } \\
\text { at } 1,020^{\circ} \mathrm{F}\end{array}$ & $\begin{array}{l}2 \text { hours } \\
\text { at } 1,200^{\circ} \mathrm{F}\end{array}$ & $\begin{array}{c}2 \text { days } \\
\text { at } 1,200^{\circ} \mathbf{F}\end{array}$ \\
\hline $\begin{array}{l}\text { None } \\
\text { Do } \\
1 / 2 \text { hour at } 1,800^{\circ} \mathrm{F}, \mathrm{W} . \mathrm{Q} \\
\text { Do } \\
\text { Do }\end{array}$ & $\begin{array}{l}\text { None } \\
1 / 2 \text { hour at } 1,600^{\circ} \mathrm{F}, \mathrm{A} . \mathrm{C} \\
\text { None } \\
1 / 2 \text { hour at } 1,600^{\circ} \mathrm{F}, \mathrm{A} . \mathrm{C} \\
2 \text { hours at } 1,600^{\circ} \mathrm{F}, \mathrm{A} . \mathrm{C}\end{array}$ & $\begin{array}{r}\mathrm{A} \\
\mathrm{C}+ \\
\mathrm{A} \\
\mathrm{E}+ \\
\mathrm{D}\end{array}$ & $\begin{array}{c}\mathrm{D} \\
\mathrm{E} \\
\mathrm{B}+ \\
\mathrm{E}+ \\
\mathrm{E}\end{array}$ & $\begin{array}{c}\mathrm{DE} \\
\mathrm{E} \\
\mathrm{D}+ \\
\mathrm{E} \\
\mathrm{E}\end{array}$ & $\begin{array}{c}\mathrm{E} \\
\mathrm{E}+ \\
\mathrm{E} \\
\mathrm{E}+ \\
\mathrm{E}\end{array}$ & $\begin{array}{r}\mathrm{E} \\
\mathrm{E} \\
\mathrm{E} \\
\mathrm{E}+ \\
\mathrm{E}\end{array}$ & $\begin{array}{r}\mathrm{E} \\
\mathrm{E}+ \\
\mathrm{E} \\
\mathrm{E} \\
\mathrm{E}+\end{array}$ & $\begin{array}{l}\mathrm{E} \\
\mathrm{D} \\
\mathrm{E} \\
\mathrm{E} \\
\mathrm{E}\end{array}$ & $\begin{array}{c}\mathrm{A} \\
\mathrm{A} \\
\mathrm{E} \\
\mathrm{E} \\
\mathrm{BC}\end{array}$ \\
\hline
\end{tabular}

TABLE 7. Effect of various treatments upon the susceptibility to intergranular attack of steel S-26

$($ Carbon $=0.068 \%$, columbium $=0.50 \% ; \mathrm{Cb} / \mathrm{C}=7.4)$

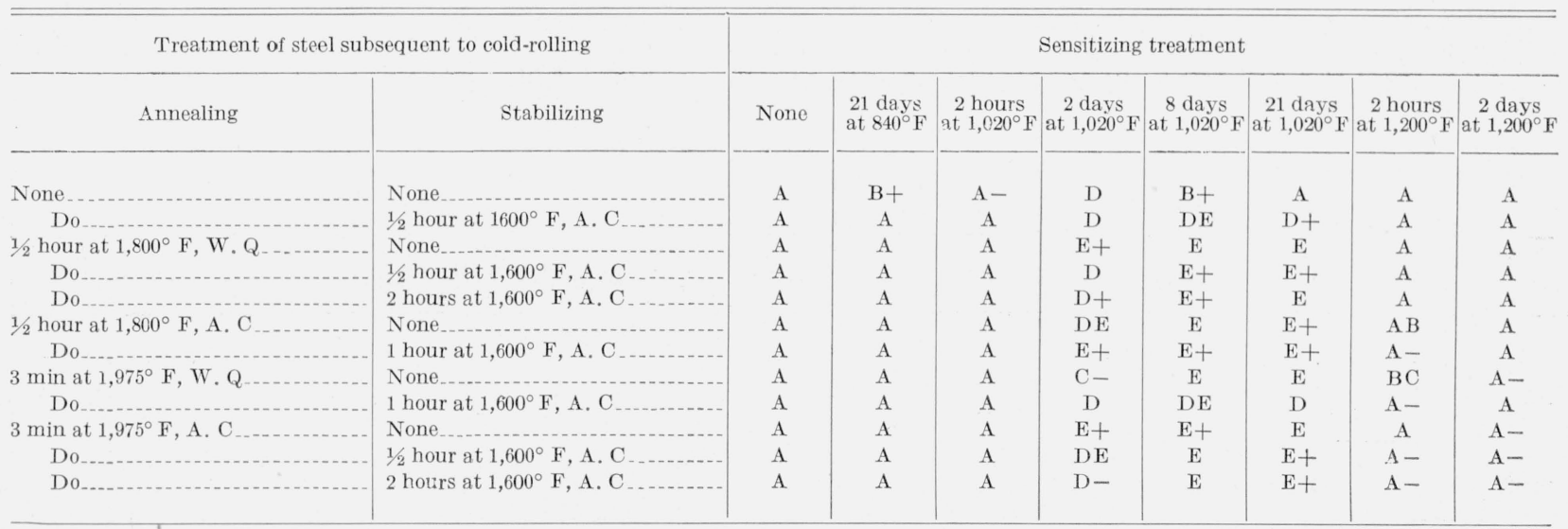


TABLE 8. Effect of various treatments upon the susceptibility to intergranular attack of steel $S-6$

(Carbon $=0.070 \%$, columbium $=0.61 \% ; \mathrm{Cb} / \mathrm{C}=8.7)$

\begin{tabular}{|c|c|c|c|c|c|c|c|c|c|}
\hline \multicolumn{2}{|c|}{ Treatment of steel subsequent to cold-rolling } & \multicolumn{8}{|c|}{ Sensitizing treatment } \\
\hline Annealing & Stabilizing & None & $\begin{array}{l}21 \text { days } \\
\text { at } 840^{\circ} \mathrm{F}\end{array}$ & $\begin{array}{c}2 \text { hours } \\
\text { at } 1,020^{\circ} \mathrm{F}\end{array}$ & $\begin{array}{c}2 \text { days } \\
\text { at } 1,020^{\circ} \mathbf{F}\end{array}$ & $\begin{array}{c}8 \text { days } \\
\text { at } 1,020^{\circ} \mathrm{F}\end{array}$ & $\begin{array}{c}21 \text { days } \\
\text { at } 1,020^{\circ} \mathrm{F}\end{array}$ & $\begin{array}{c}2 \text { hours } \\
\text { at } 1,200^{\circ} \mathrm{F}\end{array}$ & $\begin{array}{c}2 \text { days } \\
\text { at } 1,200^{\circ} \mathrm{F}\end{array}$ \\
\hline None... & None & A & B & $\mathrm{AB}$ & $\mathrm{B}+$ & $\mathrm{AB}$ & $\mathrm{AB}$ & $A-$ & A \\
\hline Do & $1 / 2$ hour at $1,600^{\circ} \mathrm{F}, \mathrm{A} . \mathrm{C} \ldots$ & A & A & A & $\mathrm{C}-$ & $\mathrm{C}-$ & B & A & A \\
\hline $1 / 2$ hour at $1,800^{\circ} \mathrm{F}, \mathrm{W}, \mathrm{Q} \ldots \ldots$ & None & A & A & A & $\mathrm{C}+$ & $\mathrm{D}-$ & $\mathrm{E}+$ & A & A \\
\hline Do & $1 / 2$ hour at $1,600^{\circ} \mathrm{F}, \mathrm{A} . \mathrm{C} \ldots$ & A & A & A & $\mathrm{D}+$ & $\mathrm{E}+$ & $\mathrm{E}+$ & A & A \\
\hline Do & 2 hours at $1,600^{\circ} \mathrm{F}, \mathrm{A} . \mathrm{C} \ldots$ & A & A & A & $\mathrm{C}-$ & $\mathrm{E}+$ & $\mathrm{D}-$ & A & A \\
\hline $1 / 2$ hour at $1,800^{\circ} \mathrm{F}, \mathrm{A} . \mathrm{C} \ldots$ & None & A & A & A & $\mathrm{BC}$ & $\mathrm{D}$ & $\mathrm{C}$ & A & A \\
\hline Do & 1 hour at $1,600^{\circ} \mathrm{F}, \mathrm{A}, \mathrm{C} \ldots$ & A & A & A & $\mathrm{D}$ & $\mathrm{D}-$ & $\mathrm{D}-$ & A & A \\
\hline $3 \mathrm{~min}$ at $1,975^{\circ} \mathrm{F}, \mathrm{W}, \mathrm{Q} \ldots$ & None & A & A & A & $\mathrm{B}+$ & $\mathrm{E}$ & $\mathrm{E}$ & A & A \\
\hline Do & 1 hour at $1,600^{\circ} \mathrm{F}, \mathrm{A} . \mathrm{C} \ldots$ & A & A & A & $\mathrm{C}$ & $\mathrm{E}$ & $\mathrm{D}$ & A & A \\
\hline $3 \mathrm{~min}$ at $1,975^{\circ} \mathrm{F}, \mathrm{A} . \mathrm{C}$ & None & A & A & A & $\mathrm{C}$ & DE & $\mathrm{E}+$ & $A-$ & $A-$ \\
\hline Do & $1 / 2$ hour at $1,600^{\circ} \mathrm{F}, \mathrm{A} . \mathrm{C} \ldots$ & A & A & A & $\mathrm{D}$ & $\mathrm{E}+$ & $\mathrm{E}$ & $A-$ & $A-$ \\
\hline Do & 2 hours at $1,600^{\circ} \mathrm{F}, \mathrm{A} . \mathrm{C} \ldots$ & $\mathrm{A}$ & A & A & $\mathrm{DE}$ & $\mathrm{DE}$ & $\mathrm{E}$ & A & $A-$ \\
\hline
\end{tabular}

TABLE 9. Effect of various treatments upon the susceptibility to intergranular attack of steel S-12

$($ Carbon $=0.074 \%$, columbium $=0,75 \% ; \mathrm{Cb} / \mathrm{C}=10.1)$

\begin{tabular}{|c|c|c|c|c|c|c|c|c|c|}
\hline \multicolumn{2}{|c|}{ Treatment of steel subsequent to cold-rolling } & \multicolumn{8}{|c|}{ Sensitizing treatment } \\
\hline Annealing & Stabilizing & None & $\begin{array}{l}21 \text { days } \\
\text { at } 840^{\circ} \mathrm{F}\end{array}$ & $\begin{array}{c}2 \text { hours } \\
\text { at } 1,020^{\circ} \mathrm{F}\end{array}$ & $\begin{array}{c}2 \text { days } \\
\text { at } 1,020^{\circ} \mathrm{F}\end{array}$ & $\begin{array}{c}8 \text { days } \\
\text { at } 1,020^{\circ} \mathrm{F}\end{array}$ & $\begin{array}{c}21 \text { days } \\
\text { at } 1,020^{\circ} \mathrm{F}\end{array}$ & $\begin{array}{l}2 \text { hours } \\
\text { at } 1,200^{\circ} \mathrm{F}\end{array}$ & $\begin{array}{c}2 \text { days } \\
\text { at } 1,200^{\circ} \mathrm{F}\end{array}$ \\
\hline None... & None & A & $\mathrm{AB}$ & $A-$ & $\mathrm{B}+$ & A & $A-$ & A & A \\
\hline Do & $1 / 2$ hour at $1,600^{\circ} \mathrm{F}, \mathrm{A} \cdot \mathrm{C} \ldots$ & A & A & A & A & A & $\mathrm{A}$ & A & $\mathrm{A}$ \\
\hline $1 / 2$ hour at $1,800^{\circ} \mathrm{F}, \mathrm{W}, \mathrm{Q} \ldots$ & None & A & A & A & A & A & $A-$ & A & A \\
\hline Do & $1 / 2$ hour at $1,600^{\circ} \mathrm{F}, \mathrm{A} . \mathrm{C} \ldots \ldots$ & A & A & $\mathrm{A}$ & A & A & $A-$ & A & A \\
\hline Do & 2 hours at $1,600^{\circ} \mathrm{F}, \mathrm{A} . \mathrm{C}$ & A & A & $\mathrm{A}$ & A & A & A & $\mathrm{A}$ & A \\
\hline $1 / 2$ hour at $1,800^{\circ} \mathrm{F}, \mathrm{A} . \mathrm{C}$ & None & A & A & A & A & $A-$ & $A-$ & A & A \\
\hline Do & 1 hour at $1,600^{\circ} \mathrm{F}, \mathrm{A} . \mathrm{C}$ & A & A & A & $\mathrm{B}+$ & B & $\mathrm{B}-$ & $A-$ & A \\
\hline $3 \mathrm{~min}$ at $1,975^{\circ} \mathrm{F}, \mathrm{W} . \mathrm{Q}$ & None & A & A & A & A & $\mathrm{B}$ & $\mathrm{D}+$ & A & A \\
\hline Do & 1 hour at $1,600^{\circ} \mathrm{F}, \mathrm{A} . \mathrm{C} \ldots$ & A & A & A & $\mathrm{B}+$ & $\mathrm{B}$ & B & A & A \\
\hline $3 \mathrm{~min}$ at $1,975^{\circ} \mathrm{F}, \mathrm{A} . \mathrm{C} \ldots$ & None & A & A & A & $A-$ & $\mathrm{AB}$ & $\mathrm{C}-$ & A & A \\
\hline Do & $1 / 2$ hour at $1,600^{\circ} \mathrm{F}, \mathrm{A} . \mathrm{C} \ldots$ & A & A & A & $\mathrm{AB}$ & $\mathrm{C}-$ & $\mathrm{C}$ & A & A \\
\hline Do & 2 hours at $1,600^{\circ} \mathrm{F}, \mathrm{A} . \mathrm{C} \ldots$ & A & A & A & $\mathrm{C}+$ & $\mathrm{C}$ & $\mathrm{C}$ & A & $A-$ \\
\hline
\end{tabular}

TABLE 10. Effect of various treatments upon the susceptibility to intergranular attack of steel $C-3$

(Carbon $=0.060 \%$, columbium $=0.71 \% ; \mathrm{Cb} / \mathrm{C}=11.8)$

\begin{tabular}{|c|c|c|c|c|c|c|c|c|c|}
\hline \multicolumn{2}{|c|}{ Treatment of steel subsequent to cold-roliing } & \multicolumn{8}{|c|}{ Sensitizing treatment } \\
\hline Annealing & Stabilizing & None & $\begin{array}{l}21 \text { days } \\
\text { at } 840^{\circ} \mathrm{F}\end{array}$ & $\begin{array}{l}2 \text { hours } \\
\text { at } 1,020^{\circ} \mathrm{F}\end{array}$ & $\begin{array}{c}2 \text { days } \\
\text { at } 1,020^{\circ} \mathrm{F}\end{array}$ & $\begin{array}{c}8 \text { days } \\
\text { at } 1,020^{\circ} \mathrm{F}\end{array}$ & $\begin{array}{l}21 \text { days } \\
\text { at } 1,020^{\circ} \mathrm{F}\end{array}$ & $\begin{array}{l}2 \text { hours } \\
\text { at } 1,200^{\circ} \mathrm{F}\end{array}$ & $\begin{array}{c}2 \text { days } \\
\text { at } 1,200^{\circ} \mathrm{F}\end{array}$ \\
\hline None.... & None_. & A & $A-$ & A & A & A & $A-$ & A & A- \\
\hline Do & $1 / 2$ hour at $1,600^{\circ} \mathrm{F}, \mathrm{A} . \mathrm{C} \ldots$ & A & A & A & A & A & A & A & A \\
\hline $1 / 2$ hour at $1,800^{\circ} \mathrm{F}, \mathrm{W} . \mathrm{Q}$ & None & A & A & A & A & A & A & A & A \\
\hline Do & $1 / 2$ hour at $1,600^{\circ} \mathrm{F}, \mathrm{A} \cdot \mathrm{C} \ldots$ & A & A & A & A & $A-$ & $A-$ & A & A \\
\hline Do & 2 hours at $1,600^{\circ} \mathrm{F}, \mathrm{A} . \mathrm{C} \ldots$ & A & A & A & A & A & A & A & A \\
\hline $1 / 2$ hour at $1,800^{\circ} \mathrm{F}, \mathrm{A}$. C & None & A & A & A & A & A & A & A & A \\
\hline Do & 1 hour at $1,600^{\circ} \mathrm{F}, \mathrm{A} . \mathrm{C} \ldots$ & A & A & A & A & $A-$ & $A-$ & $A-$ & A \\
\hline $3 \min$ at $1,975^{\circ} \mathrm{F}, \mathrm{W} . \mathrm{Q}$ - & None & A & A & A & A & A & A & A & A \\
\hline Do & 1 hour at $1,600^{\circ} \mathrm{F}, \mathrm{A} . \mathrm{C} \ldots \ldots$ & A & A & A & A & A & A & A & A \\
\hline $3 \mathrm{~min}$ at $1,975^{\circ} \mathrm{F}, \mathrm{A} . \mathrm{C}$ & None & A & A & A & A & A & A & $A-$ & A \\
\hline Do & $1 / 2$ hour at $1,600^{\circ} \mathrm{F}, \mathrm{A} . \mathrm{C} \ldots$ & A & A & A & A & A & A & A & A \\
\hline Do & 2 hours at $1,600^{\circ} \mathrm{F}, \mathrm{A} . \mathrm{C} \ldots$ & A & A & A & A & A & A & A & A \\
\hline
\end{tabular}


TABLE 11. Effect of various treatments upon the susceptibility to intergranular attack of steel S-27

(Carbon $=0.087 \%$, columbium $=0.76 \% ; \mathrm{Cb} / \mathrm{C}=8.7$ )

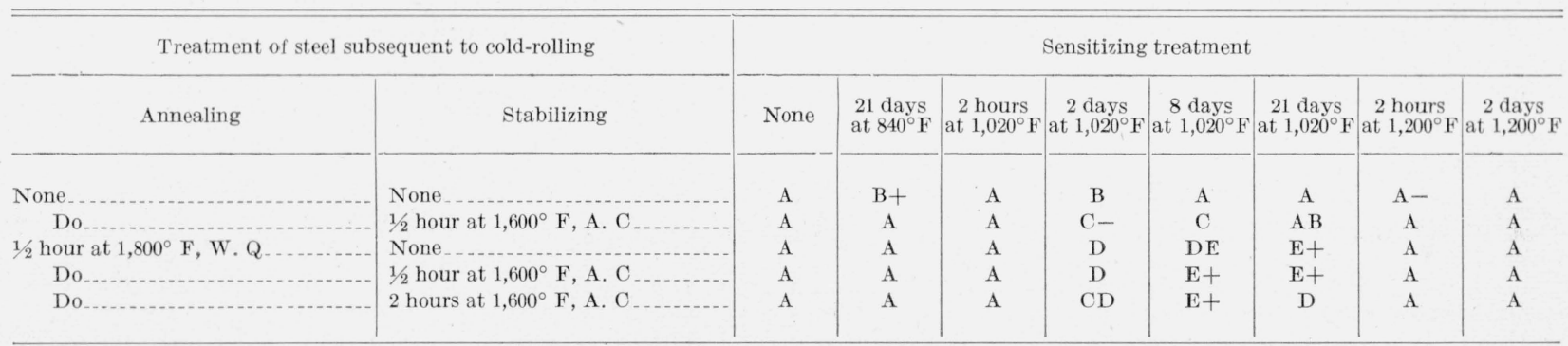

TABLE 12. Effect of various treatments upon the susceptibility to intergranular attack of steel S-28

(Carbon $=0.115 \%$, columbium $=0.92 \% ; \mathrm{Cb} / \mathrm{C}=8.0$ )

\begin{tabular}{|c|c|c|c|c|c|c|c|c|c|}
\hline \multicolumn{2}{|c|}{ Treatment of steel subsequent to cold-rolling } & \multicolumn{8}{|c|}{ Sensitizing treatment } \\
\hline Annealing & Stabilizing & None & $\begin{array}{l}21 \text { days } \\
\text { at } 840^{\circ} \mathrm{F}\end{array}$ & $\begin{array}{c}2 \text { hours } \\
\text { at } 1,020^{\circ} \mathrm{F}\end{array}$ & $\begin{array}{c}2 \text { days } \\
\text { at } 1,020^{\circ} \mathrm{F}\end{array}$ & $\begin{array}{c}8 \text { days } \\
\text { at } 1,020^{\circ} \mathrm{F}\end{array}$ & $\begin{array}{c}21 \text { days } \\
\text { at } 1,020^{\circ} \mathrm{F}\end{array}$ & $\begin{array}{c}2 \text { hours } \\
\text { at } 1,200^{\circ} \mathrm{F}\end{array}$ & $\begin{array}{c}2 \text { days } \\
\text { at } 1,200^{\circ} \mathrm{F}\end{array}$ \\
\hline None.... & None & A & B & $A-$ & $\mathrm{C}-$ & $\mathrm{B}+$ & $A-$ & $A-$ & A \\
\hline Do & $1 / 2$ hour at $1,600^{\circ} \mathrm{F}, \mathrm{A} . \mathrm{C}$ & A & A & A & $\mathrm{D}+$ & $\mathrm{D}+$ & $\mathrm{BC}$ & A & A \\
\hline $1 / 2$ hour at $1,800^{\circ} \mathrm{F}, \mathrm{W}, \mathrm{Q} \ldots$ & None & A & A & A & $\mathrm{DE}$ & $\mathrm{E}$ & $\mathrm{E}$ & A & A \\
\hline Do & $1 / 2$ hour at $1,600^{\circ} \mathrm{F}, \mathrm{A}, \mathrm{C} \ldots$ & A & A & A & D & $\mathrm{E}+$ & $\mathrm{E}+$ & A & A \\
\hline Do & 2 hours at $1,600^{\circ} \mathrm{F}, \mathrm{A} . \mathrm{C} \ldots$ & A & A & A & $\mathrm{C}-$ & $\mathrm{E}+$ & DE & A & A \\
\hline $1 / 2$ hour at $1,800^{\circ} \mathrm{F}, \mathrm{A} . \mathrm{C}$ & None & A & A & A & $\mathrm{DE}$ & DE & $\mathrm{E}+$ & $A-$ & A \\
\hline Do & 1 hour at $1,600^{\circ} \mathrm{F}, \mathrm{A} . \mathrm{C} \ldots$ & A & A & A & $\mathrm{D}$ & DE & $\mathrm{D}-$ & $\mathrm{B}+$ & A \\
\hline $3 \mathrm{~min}$ at $1,975^{\circ} \mathrm{F}, \mathrm{W} \cdot \mathrm{Q}$ & None & A & A & A & $\mathrm{D}+$ & $\mathrm{E}$ & $\mathrm{E}$ & $\mathrm{B}-$ & A \\
\hline Do & 1 hour at $1,600^{\circ} \mathrm{F}, \mathrm{A} . \mathrm{C} \ldots$ & A & A & A & $\mathrm{CD}$ & $\mathrm{E}$ & $\mathrm{C}-$ & $A-$ & A \\
\hline $3 \mathrm{~min}$ at $1,975^{\circ} \mathrm{F}, \mathrm{A}, \mathrm{C} \ldots$ & None & A & A & A & $\mathrm{E}+$ & $\mathrm{DE}$ & $\mathrm{E}$ & $A-$ & $\mathrm{A}$ \\
\hline Do & $1 / 2$ hour at $1,600^{\circ} \mathrm{F}, \mathrm{A}, \mathrm{C}$ & A & A & A & $\mathrm{D}-$ & $\mathbf{E}+$ & $\mathbf{E}+$ & $A-$ & A \\
\hline Do & 2 hours at $1,600^{\circ} \mathrm{F}, \mathrm{A}, \mathrm{C} \ldots$ & A & A & A & $\mathrm{E}+$ & $\mathrm{E}+$ & $\mathrm{DE}$ & $A-$ & $A-$ \\
\hline
\end{tabular}

TABLE 13. Effect of various treatments upon the susceptibility to intergranular attack of steel $S-17$

(Carbon $=0.117 \%$, columbium $=1.14 \% ; \mathrm{Cb} / \mathrm{C}=9.7)$

\begin{tabular}{|c|c|c|c|c|c|c|c|c|c|}
\hline \multicolumn{2}{|c|}{ Treatment of steel subsequent to cold-rolling } & \multicolumn{8}{|c|}{ Sensitizing treatment } \\
\hline Annealing & Stabilizing & None & $\begin{array}{l}21 \text { days } \\
\text { at } 840^{\circ} \mathrm{F}\end{array}$ & $\begin{array}{l}2 \text { hours } \\
\text { at } 1,020^{\circ} \mathrm{F}\end{array}$ & $\begin{array}{c}2 \text { days } \\
\text { at } 1,020^{\circ} \mathrm{F}\end{array}$ & $\begin{array}{c}8 \text { days } \\
\text { at } 1,020^{\circ} \mathrm{F}\end{array}$ & $\begin{array}{c}21 \text { days } \\
\text { at } 1,020^{\circ} \mathrm{F}\end{array}$ & $\begin{array}{l}2 \text { hours } \\
\text { at } 1,200^{\circ} \mathrm{F}\end{array}$ & $\begin{array}{c}2 \text { days } \\
\text { at } 1,200^{\circ} \mathrm{F}\end{array}$ \\
\hline None. - & None & A & $\mathrm{B}+$ & $A-$ & $\mathrm{AB}$ & $\mathrm{AB}$ & $A-$ & A & A \\
\hline Do & $1 / 2$ hour at $1,600^{\circ} \mathrm{F}, \mathrm{A}$. C & A & A & A & A & A & A & A & A \\
\hline $1 / 2$ hour at $1,800^{\circ} \mathrm{F}, \mathrm{W}$. & None & A & A & A & A & A & $\mathrm{B}+$ & A & A \\
\hline Do & $1 / 2$ hour at $1,600^{\circ} \mathrm{F}, \mathrm{A} . \mathrm{C}$ & A & A & A & $\mathrm{AB}$ & B & $A B$ & A & A \\
\hline Do & 2 hours at $1,600^{\circ} \mathrm{F}, \mathrm{A} . \mathrm{C}$ & A & A & A & A & A & A & A & A \\
\hline $1 / 2$ hour at $1,800^{\circ} \mathrm{F}, \mathrm{A}$. C & None & A & A & $\mathrm{A}$ & A & $\mathrm{AB}$ & A & A & $A$ \\
\hline Do & 1 hour at $1,600^{\circ} \mathrm{F}, \mathrm{A}, \mathrm{C}$ & A & A & A & $\mathrm{BC}$ & $\mathrm{BC}$ & $\mathrm{B}+$ & A & A \\
\hline $3 \mathrm{~min}$ at $1,975^{\circ} \mathrm{F}, \mathrm{W}, \mathrm{Q}$ & None & A & A & A & $A-$ & $\mathrm{C}+$ & $\mathrm{D}+$ & A & A \\
\hline Do & 1 hour at $1,600^{\circ} \mathrm{F}, \mathrm{A}, \mathrm{C}$ & A & A & A & $\mathrm{B}+$ & B & B & A & A \\
\hline $3 \mathrm{~min}$ at $1,975^{\circ} \mathrm{F}, \mathrm{A} . \mathrm{C}$ & None & A & A & A & $A-$ & $B-$ & $\mathrm{CD}$ & A & A \\
\hline Do & $1 / 2$ hour at $1,600^{\circ} \mathrm{F}, \mathrm{A}$. C & A & A & A & $\mathrm{B}+$ & $\mathrm{D}$ & $\mathrm{C}$ & $A-$ & A \\
\hline Do & 2 hours at $1,600^{\circ} \mathrm{F}, \mathrm{A}$. C & A & A & A & $\mathrm{C}-$ & $\mathrm{C}$ & $\mathrm{C}$ & $\mathrm{A}-$ & $A-$ \\
\hline
\end{tabular}


TABLE 14. Effect of various treatments upon the susceptibitity to intergranular attack of steel S-18

$($ Carbon $=0.132 \%$, columbium $=1.47 \% ; \mathrm{Cb} / \mathrm{C}=11.1)$

\begin{tabular}{|c|c|c|c|c|c|c|c|c|c|}
\hline \multicolumn{2}{|c|}{ Treatment of steel subsequent to cold-rolling } & \multicolumn{8}{|c|}{ Sensitizing treatment } \\
\hline Annealing & Stabilizing & None & $\begin{array}{l}21 \text { days } \\
\text { at } 840^{\circ} \mathrm{F}\end{array}$ & $\begin{array}{l}2 \text { hours } \\
\text { at } 1,020^{\circ} \mathrm{F}\end{array}$ & $\begin{array}{c}2 \text { days } \\
\text { at } 1,020^{\circ} \mathbf{F}\end{array}$ & $\begin{array}{c}8 \text { days } \\
\text { at } 1,020^{\circ} \mathrm{F}\end{array}$ & $\begin{array}{l}21 \text { days } \\
\text { at } 1,020^{\circ} \mathrm{F}\end{array}$ & $\begin{array}{c}2 \text { hours } \\
\text { at } 1,200^{\circ} \mathrm{F}\end{array}$ & $\begin{array}{c}2 \text { days } \\
\text { at } 1,200^{\circ} \mathrm{F}\end{array}$ \\
\hline None_... & None & A & A & A & $A-$ & $A-$ & $A-$ & $A-$ & $\mathrm{A}$ \\
\hline Do & $1 / 2$ hour at $1,600^{\circ} \mathrm{F}, \mathrm{A} . \mathrm{C} \ldots$ & A & A & A & A & A & A & A & A \\
\hline $1 / 2$ hour at $1,800^{\circ} \mathrm{F}, \mathrm{W} . \mathrm{Q} \ldots$ & None & A & A & A & A & A & $A-$ & A & A \\
\hline Do & $1 / 2$ hour at $1,600^{\circ} \mathrm{F}, \mathrm{A} . \mathrm{C} \ldots \ldots$ & A & A & A & A & A & $A-$ & A & A \\
\hline Do & 2 hours at $1,600^{\circ} \mathrm{F}, \mathrm{A} . \mathrm{C} \ldots \ldots$ & A & A & A & A & A & A & $\mathrm{A}$ & A \\
\hline $1 / 2$ hour at $1,800^{\circ} \mathrm{F}, \mathrm{A}$. C $\ldots$ & None $\ldots \ldots \ldots$ & A & A & A & A & A & A & A & A \\
\hline Do & 1 hour at $1,600^{\circ} \mathrm{F}, \mathrm{A} . \mathrm{C} \ldots$ & A & A & A & $A-$ & $\mathrm{B}+$ & $A-$ & A & A \\
\hline $3 \mathrm{~min}$ at $1,975^{\circ} \mathrm{F}, \mathrm{W}, \mathrm{Q} \ldots \ldots$ & None & A & A & A & A & A & B & A & A \\
\hline Do $\ldots$ & 1 hour at $1,600^{\circ} \mathrm{F}, \mathrm{A}, \mathrm{C} \ldots \ldots$ & A & A & A & A & $A-$ & $A-$ & A & A \\
\hline $3 \mathrm{~min}$ at $1,975^{\circ} \mathrm{F}, \mathrm{A} . \mathrm{C} \ldots$ & None....... & A & A & A & $A-$ & $A-$ & $\mathrm{B}+$ & A & A \\
\hline Do & $1 / 2$ hour at $1,600^{\circ} \mathrm{F}, \mathrm{A} . \mathrm{C} \ldots$ & A & A & $\mathrm{A}$ & A & $\mathrm{B}+$ & $\mathrm{B}$ & A & A \\
\hline Do _. & 2 hours at $1,600^{\circ} \mathrm{F}, \mathrm{A} . \mathrm{C} \ldots$ & A & A & A & A & $A-$ & $A-$ & A & A \\
\hline
\end{tabular}

TABLE 15. Effect of various treatments upon the susceptibility to intergranular attack of steel S-34

$($ Carbon $=0.067 \%$, titanium $=0.26 \% ; \mathrm{Ti} / \mathrm{C}=3.9)$

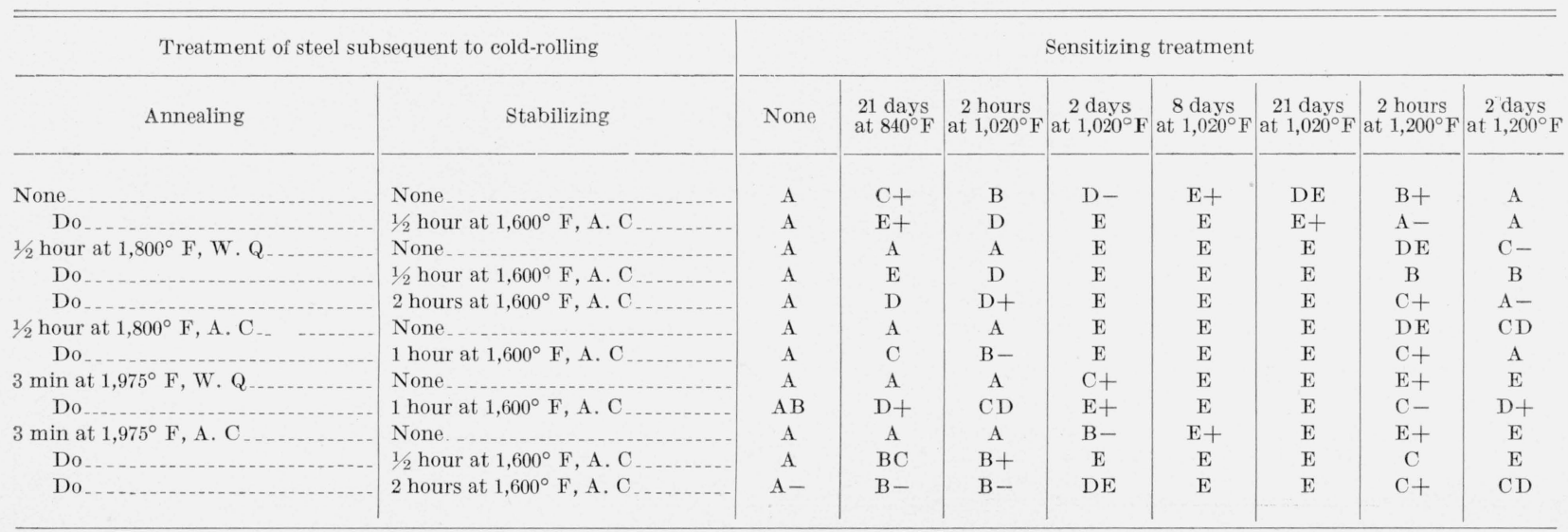

TABLE 16. Effect of various treatments upon the susceptibility to intergranular attack of steel S-21

$($ Carbon $=0.071 \%$, titanium $=0.32 \% ; \mathrm{Ti} / \mathrm{C}=4.5)$

\begin{tabular}{|c|c|c|c|c|c|c|c|c|c|}
\hline \multicolumn{2}{|c|}{ Treatment of steel subsequent to cold-rolling } & \multicolumn{8}{|c|}{ Sensitizing treatment } \\
\hline Annealing & Stabilizing & None & $\begin{array}{l}21 \text { days } \\
\text { at } 840^{\circ} \mathrm{F}\end{array}$ & $\begin{array}{l}2 \text { hours } \\
\text { at } 1,020^{\circ} \mathrm{F}\end{array}$ & $\begin{array}{c}2 \text { days } \\
\text { at } 1,020^{\circ} \mathrm{F}\end{array}$ & $\begin{array}{c}8 \text { days } \\
\text { at } 1,020^{\circ} \mathrm{F}\end{array}$ & $\begin{array}{c}21 \text { days } \\
\text { at } 1,020^{\circ} \mathrm{F}\end{array}$ & $\begin{array}{c}2 \text { hours } \\
\text { at } 1,200^{\circ} \mathrm{F}\end{array}$ & $\begin{array}{c}2 \text { days } \\
\text { at } 1.200^{\circ} \mathrm{F}\end{array}$ \\
\hline None..... & None & A & $\mathrm{C}-$ & $\mathrm{B}$ & $\mathrm{D}$ & $\mathrm{DE}$ & $\mathrm{D}$ & $\mathrm{B}+$ & $\mathrm{A}$ \\
\hline Do & $1 / 2$ hour at $1,600^{\circ} \mathrm{F}, \mathrm{A}, \mathrm{C}$ & A & $A B$ & $\mathrm{AB}$ & $\mathrm{E}+$ & $\mathrm{E}+$ & $\mathrm{E}+$ & $A-$ & A \\
\hline $1 / 2$ hour at $1,800^{\circ} \mathrm{F}, \mathrm{W} \cdot \mathrm{Q} \ldots$ & None & A & A & A & $\mathrm{E}$ & $\mathrm{E}$ & $\mathrm{E}$ & $\mathrm{D}$ & $A-$ \\
\hline Do & $1 / 2$ hour at $1,600^{\circ} \mathrm{F}, \mathrm{A}, \mathrm{C} \ldots$ & A & $\mathrm{CD}$ & B & $\mathrm{E}$ & $\mathrm{E}$ & $\mathrm{E}$ & A & A \\
\hline Do & 2 hours at $1,600^{\circ} \mathrm{F}, \mathrm{A}, \mathrm{C} \ldots$ & A & $A-$ & $\mathrm{AB}$ & $\mathrm{E}+$ & $\mathrm{E}+$ & $\mathrm{E}$ & $\mathrm{AB}$ & $A-$ \\
\hline $1 / 2$ hour at $1,800^{\circ} \mathrm{F}, \mathrm{A} . \mathrm{C} \ldots$ & None & A & A & A & $\mathrm{E}$ & $\mathrm{E}$ & $\mathrm{E}$ & $\mathrm{D}+$ & $\mathrm{B}+$ \\
\hline Do & 1 hour at $1,600^{\circ} \mathrm{F}, \mathrm{A} . \mathrm{C} \ldots$ & A & $\mathrm{B}+$ & $A-$ & $\mathrm{CD}$ & $\mathrm{D}-$ & $\mathrm{D}$ & $\mathrm{BC}$ & A \\
\hline $3 \mathrm{~min}$ at $1,975^{\circ} \mathrm{F}, \mathrm{W} . \mathrm{Q} \ldots$ & None & A & A & A & $\mathrm{D}+$ & $\mathrm{E}$ & $\mathrm{E}$ & $\mathrm{E}$ & $\mathrm{DE}$ \\
\hline Do & 1 hour at $1,600^{\circ} \mathrm{F}, \mathrm{A} . \mathrm{C} \ldots$ & A & $\mathrm{BC}$ & $\mathrm{B}+$ & $\mathrm{E}+$ & $\mathrm{E}$ & $\mathrm{E}$ & $\mathrm{B}-$ & B \\
\hline $3 \mathrm{~min}$ at $1,975^{\circ} \mathrm{F}, \mathrm{A} . \mathrm{C} \ldots$ & None & A & A & A & $\mathrm{DE}$ & $\mathrm{E}+$ & $\mathrm{E}+$ & $\mathrm{DE}$ & $\mathrm{E}+$ \\
\hline Do & $1 / 2$ hour at $1,600^{\circ} \mathrm{F}, \mathrm{A} . \mathrm{C} \ldots$ & A & $\mathrm{AB}$ & $\mathrm{AB}$ & $\mathrm{E}+$ & $\mathrm{E}$ & $\mathrm{E}$ & $\mathrm{BC}$ & $\mathrm{CD}$ \\
\hline Do & 2 hours at $1,600^{\circ} \mathrm{F}, \mathrm{A} . \mathrm{C} \ldots$ & $A-$ & $A-$ & $\mathrm{B}$ & $\mathrm{D}-$ & $\mathrm{E}$ & $\mathrm{E}$ & $\mathrm{BC}$ & $\mathrm{C}$ \\
\hline
\end{tabular}


TABLE 17. Effect of various treatments upon the susceptibility to intergranular attack of steel S-39

$($ Carbon $=0.065 \%$, titanium $=0.36 \% ; \mathrm{Ti} / \mathrm{C}=5.5)$

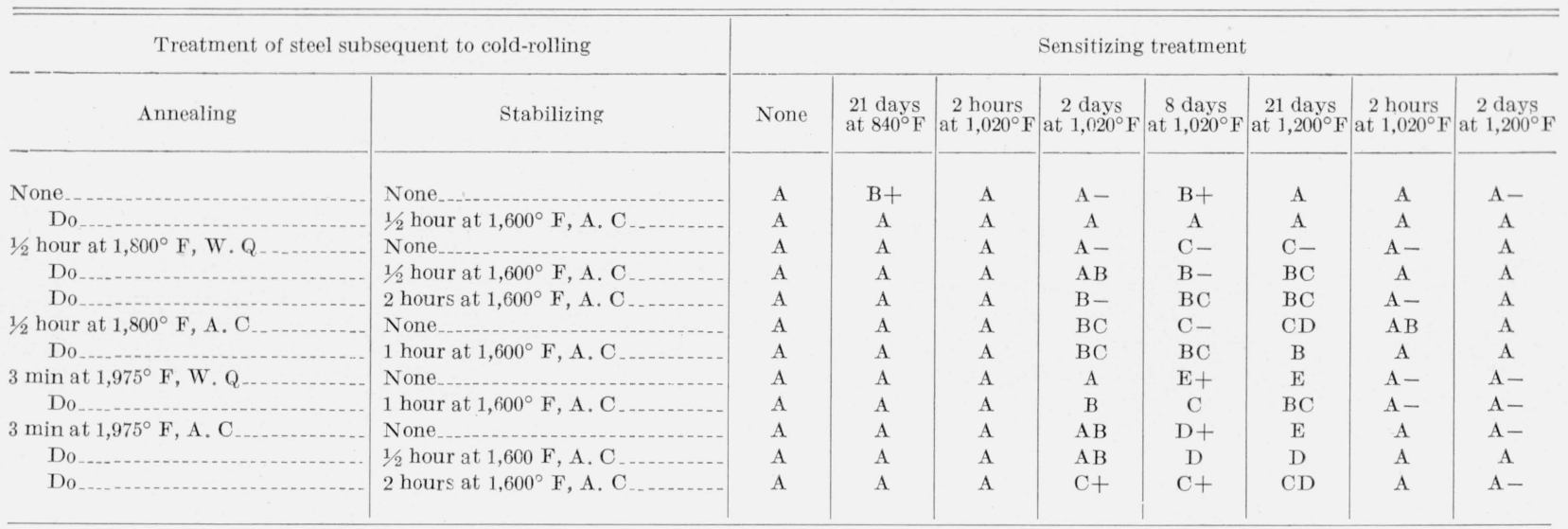

TABLE 18. Effect of various treatments upon the susceptibility to intergranular attack of steel $C-6$

(Carbon $=0.064 \%$, titanium $=0.35 \%, \mathrm{Ti} / \mathrm{C}=5.5)$

\begin{tabular}{|c|c|c|c|c|c|c|c|c|c|}
\hline Annealing & Stabilizing & None & $\begin{array}{l}21 \text { days } \\
\text { at } 840^{\circ} \mathrm{F}\end{array}$ & $\begin{array}{l}2 \text { hours } \\
\text { at } 1,020^{\circ} \mathrm{F}\end{array}$ & $\begin{array}{c}2 \text { days } \\
\text { at } 1,020^{\circ} \mathrm{F}\end{array}$ & $\begin{array}{c}8 \text { days } \\
\text { at } 1,020^{\circ} \mathrm{F}\end{array}$ & $\begin{array}{l}21 \text { days } \\
\text { at } 1,020^{\circ} \mathrm{F}\end{array}$ & $\begin{array}{c}2 \text { hours } \\
\text { at } 1,200^{\circ} \mathrm{F}\end{array}$ & $\begin{array}{c}2 \text { days } \\
\text { at } 1,200^{\circ} \mathrm{F}\end{array}$ \\
\hline None.... & None & A & A & A & A & A- & A & A & A \\
\hline Do & $1 / 2$ hour at $1,600^{\circ} \mathrm{F}, \mathrm{A}, \mathrm{C}$ & A & A & A & A & A & A & A & A \\
\hline $1 / 2$ hour at $1,800^{\circ} \mathrm{F}, \mathrm{W} \cdot \mathrm{Q}$ & None & A & A & A & $A-$ & $\mathrm{AB}$ & $\mathrm{C}-$ & A & A \\
\hline Do & 1 hour at $1,600^{\circ} \mathrm{F}, \mathrm{A} . \mathrm{C}$ & A & A & $\mathrm{A}$ & $A-$ & $\mathrm{BC}$ & $\mathrm{B}-$ & $\mathrm{A}$ & A \\
\hline 3 -min at $1,975^{\circ} \mathrm{F}, \mathrm{W}, \mathrm{Q}$ & None & A & $\mathrm{A}$ & A & $\mathrm{A}$ & $\mathrm{D}$ & $\mathrm{D}-$ & A & $A-$ \\
\hline Do & 1 hour at $1,600^{\circ} \mathrm{F}, \mathrm{A}, \mathrm{C}$ & A & $\mathrm{A}$ & A & $\mathrm{AB}$ & B & $\mathrm{C}-$ & A & A \\
\hline 3 -min at $1,975^{\circ} \mathrm{F}, \mathrm{A} . \mathrm{C}$ & None & A & A & A & $\mathrm{A}$ & $\mathrm{D}+$ & $\mathrm{E}+$ & $A$ & $\mathrm{~B}$ \\
\hline Do & $1 / 2$ hour at $1,600^{\circ} \mathrm{F}, \mathrm{A} . \mathrm{C}$. & A & A & A & $\mathrm{B}+$ & $\mathrm{CD}$ & $\mathrm{D}$ & A & A- \\
\hline Do ............. & 2 hours at $1,600^{\circ} \mathrm{F}, \mathrm{A} . \mathrm{C}$ & A & A & A & $\mathrm{C}+$ & $\mathrm{C}+$ & $\mathrm{C}+$ & A & $A-$ \\
\hline
\end{tabular}

TABLE 19. Effect of various treatments upon the susceptibitity to intergranular attack of steel C-2

$($ Carbon $=0.070 \%$, titanium $=0.50 \% ; \mathrm{Ti} / \mathrm{C}=7.1)$

\begin{tabular}{|c|c|c|c|c|c|c|c|c|c|}
\hline \multicolumn{2}{|c|}{ Treatment of steel subsequent to cold-rolling } & \multicolumn{8}{|c|}{ Sensitizing treatment } \\
\hline Annealing & Stabilizing & None & $\begin{array}{l}21 \text { days } \\
\text { at } 840^{\circ} \mathrm{F}\end{array}$ & $\begin{array}{c}2 \text { hours } \\
\text { at } 1,020^{\circ} \mathrm{F}\end{array}$ & $\begin{array}{c}2 \text { days } \\
\text { at } 1,020^{\circ} \mathrm{F}\end{array}$ & $\begin{array}{c}8 \text { days } \\
\text { at } 1,020^{\circ} \mathrm{F}\end{array}$ & $\begin{array}{c}21 \text { days } \\
\text { at } 1,020^{\circ} \mathrm{F}\end{array}$ & $\begin{array}{c}2 \text { hours } \\
\text { at } 1,200^{\circ} \mathrm{F}\end{array}$ & $\begin{array}{c}2 \text { days } \\
\text { at } 1,200^{\circ} \mathrm{F}\end{array}$ \\
\hline None ........ & None & A & $\mathrm{C}-$ & A & $\mathrm{AB}$ & A & $\mathrm{B}-$ & A & A \\
\hline Do & $1 / 2$ hour at $1,600^{\circ} \mathrm{F}, \mathrm{A} . \mathrm{C}$ & A & A & A & A & A & A & A & A \\
\hline $1 / 2$ hour at $1,800^{\circ} \mathrm{F}, \mathrm{W} . \mathrm{Q}$ & None & A & A & $\mathrm{A}$ & A & A & A & A & A \\
\hline Do & $1 / 2$ hour at $1,600^{\circ} \mathrm{F}, \mathrm{A} . \mathrm{C}$ & A & A & A & A & A & $\mathrm{A}$ & A & A \\
\hline Do & 2 hours at $1,600^{\circ} \mathrm{F}, \mathrm{A} . \mathrm{C}$ & $\mathrm{A}$ & A & A & A & A & A & A & A \\
\hline $1 / 2$ hour at $1,800^{\circ} \mathrm{F}, \mathrm{A} \cdot \mathrm{C} \ldots$ & None & A & A & A & A & $\mathrm{B}+$ & $\mathrm{B}+$ & A & A \\
\hline Do & 1 hour at $1,600^{\circ} \mathrm{F}, \mathrm{A} . \mathrm{C} \ldots \ldots$ & A & A & A & A & A & $A-$ & $A-$ & A \\
\hline $3 \mathrm{~min}$ at $1,975^{\circ} \mathrm{F}, \mathrm{W} . \mathrm{Q} \ldots$ & None & A & A & A & A & $\mathrm{BC}$ & $\mathrm{D}$ & A & A \\
\hline Do & 1 hour at $1,600^{\circ} \mathrm{F}, \mathrm{A} . \mathrm{C} \ldots$ & A & A & A & A & A & $A-$ & A & A \\
\hline $3 \mathrm{~min}$ at $1,975^{\circ} \mathrm{F}, \mathrm{A} . \mathrm{C}$ & None & A & A & A & $\mathrm{A}$ & $\mathrm{B}+$ & $\mathrm{D}+$ & A & A \\
\hline Do & $1 / 2$ hour at $1,600^{\circ} \mathrm{F}, \mathrm{A} . \mathrm{C}$ & A & A & A & A & $A-$ & $A-$ & A & $A-$ \\
\hline Do & 2 hours at $1,600^{\circ} \mathrm{F}, \mathrm{A} . \mathrm{C} \ldots \ldots$ & $A-$ & $A-$ & A & A & $A-$ & A & A & A \\
\hline
\end{tabular}


TABLE 20. Effect of various treatments upon the susceptibility to intergranular attack of steel $C-8$

$($ Carbon $=0.075 \%$, titanium $=0.59 \% ; \mathrm{Ti} / \mathrm{C}=7.9)$

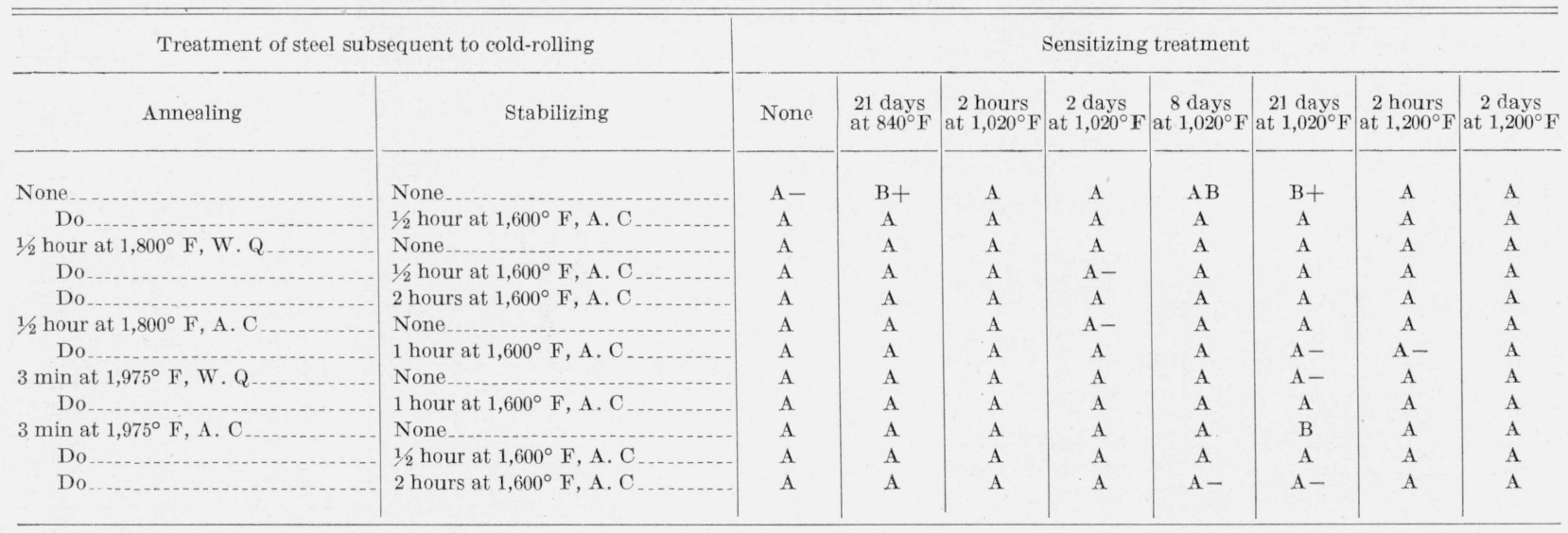

Table 21. Effect of various treatments upon the susceptibitity to intergranular attack of steel S-25

(Carbon $=0.082 \%$, titanium $=0.37 \% ; \mathrm{Ti} / \mathrm{C}=4.5)$

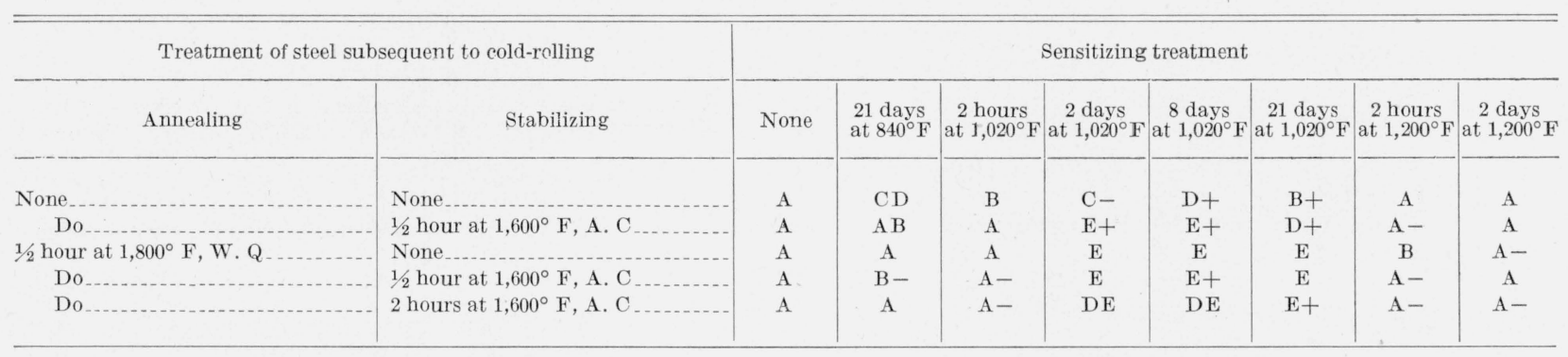

TABLE 22. Effect of various treatments upon the susceptibility to intergranular attack of steel S-35

$($ Carbon $=0.107 \%$, titanium $=0.44 \% ; \mathrm{Ti} / \mathrm{C}=4.1)$

\begin{tabular}{|c|c|c|c|c|c|c|c|c|c|}
\hline \multicolumn{2}{|c|}{ Treatment of steel subsequent to cold-rolling. } & \multicolumn{8}{|c|}{ Sensitizing treatment } \\
\hline Annealing & Stabilizing & None & $\begin{array}{l}21 \text { days } \\
\text { at } 840^{\circ} \mathrm{F}\end{array}$ & $\begin{array}{l}2 \text { hours } \\
\text { at } 1,020^{\circ} \mathrm{F}\end{array}$ & $\begin{array}{c}2 \text { days } \\
\text { at } 1,020^{\circ} \mathbf{F}\end{array}$ & $\begin{array}{c}8 \text { days } \\
\text { at } 1,020^{\circ} \mathrm{F}\end{array}$ & $\begin{array}{l}21 \text { days } \\
\text { at } 1,020^{\circ} \mathrm{F}\end{array}$ & $\begin{array}{l}2 \text { hours } \\
\text { at } 1,200^{\circ} \mathrm{F}\end{array}$ & $\begin{array}{c}2 \text { days } \\
\text { at } 1,200^{\circ} \mathrm{F}\end{array}$ \\
\hline None..... & None__._. & A & $\mathrm{E}+$ & $\mathrm{D}+$ & DE & $\mathrm{C}+$ & $\mathrm{B}$ & A & A \\
\hline Do & $1 / 2$ hour at $1,600^{\circ} \mathrm{F}, \mathrm{A} . \mathrm{C}$. & A & $\mathrm{AB}$ & $A-$ & DE & $\mathrm{E}$ & $\mathrm{D}$ & $A-$ & A \\
\hline $1 / 2$ hour at $1,800^{\circ} \mathrm{F}, \mathrm{W} \cdot \mathrm{Q}$ & None & A & A & A & $\mathrm{E}$ & $\mathrm{E}$ & $\mathrm{E}$ & $\mathrm{D}$ & A \\
\hline Do & $1 / 2$ hour at $1,600^{\circ} \mathrm{F}, \mathrm{A} . \mathrm{C}$. & A & $\mathrm{C}$ & $\mathrm{B}$ & $\mathrm{E}$ & $\mathrm{E}$ & $\mathrm{E}$ & A & A \\
\hline Do & 2 hours at $1,600^{\circ} \mathrm{F}, \mathrm{A} . \mathrm{C}$. & A & A & A & $\mathrm{DE}$ & $\mathrm{E}+$ & $\mathrm{E}$ & $\mathrm{AB}$ & $A-$ \\
\hline $1 / 2$ hour at $1,800^{\circ} \mathrm{F}, \mathrm{A} . \mathrm{C}$ & None__._. & A & A & A & $\mathrm{D}-$ & $\mathrm{E}$ & $\mathrm{DE}$ & $\mathrm{B}-$ & A \\
\hline Do & 1 hour at $1,600^{\circ} \mathrm{F}, \mathrm{A} . \mathrm{C}_{-}$ & A & $\mathrm{B}+$ & $A-$ & $\mathrm{D}$ & $\mathrm{DE}$ & $\mathrm{D}$ & $\mathrm{BC}$ & A \\
\hline $3 \mathrm{~min}$ at $1,975^{\circ} \mathrm{F}, \mathrm{W} . \mathrm{Q}$ & None & A & A & A & $\mathrm{C}-$ & $\mathrm{E}$ & $\mathrm{E}$ & $\mathrm{E}$ & DE \\
\hline Do & 1 hour at $1,600^{\circ} \mathrm{F}, \mathrm{A} . \mathrm{C}_{-}$ & A & $\mathrm{B}+$ & $\mathrm{AB}$ & $\mathrm{D}-$ & $\mathrm{E}$ & $\mathrm{E}$ & $\mathrm{B}-$ & $\mathrm{AB}$ \\
\hline $3 \mathrm{~min}$ at $1,975^{\circ} \mathrm{F}, \mathrm{A} . \mathrm{C}$ & None _._. & $\mathrm{A}$ & A & A & $\mathrm{DE}$ & $\mathrm{E}$ & $\mathrm{E}$ & $\mathrm{D}-$ & $\mathrm{E}+$ \\
\hline Do & $1 / 2$ hour at $1,600^{\circ} \mathrm{F}, \mathrm{A}$. C. & A & $\mathrm{B}$ & $\mathrm{A}$ & $\mathrm{DE}$ & $\mathrm{E}$ & $\mathrm{E}$ & $\mathrm{B}+$ & $\mathrm{C}+$ \\
\hline Do $\ldots . . .$. & 2 hours at $1,600^{\circ} \mathrm{F}, \mathrm{A} . \mathrm{C}_{-}$ & A & $\mathrm{B}+$ & $\mathrm{B}$ & $\mathrm{D}-$ & $\mathrm{E}$ & $\mathrm{E}$ & $\mathrm{B}$ & $\mathrm{C}+$ \\
\hline
\end{tabular}


TABLE 23. Effect of various treatments upon the susceptibility to intergranular attack of steel S-36

$($ Carbon $=0.109 \%$, titanium $=0.54 \% ; \mathrm{Ti} / \mathrm{C}=5.0)$

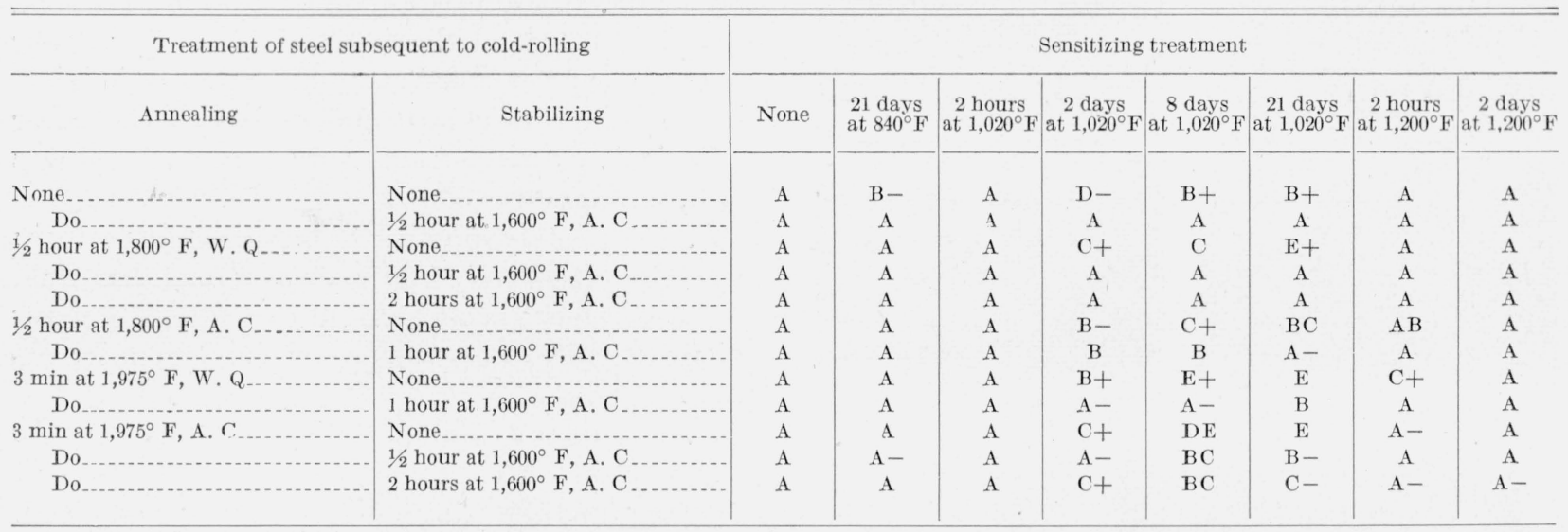

TABLE 24. Effect of various treatments upon the susceptibility of intergranular attack of steel S-32

(Carbon $=0.105 \%$, titanium $=0.61 \% ; \mathrm{Ti} / \mathrm{C}=5.8)$

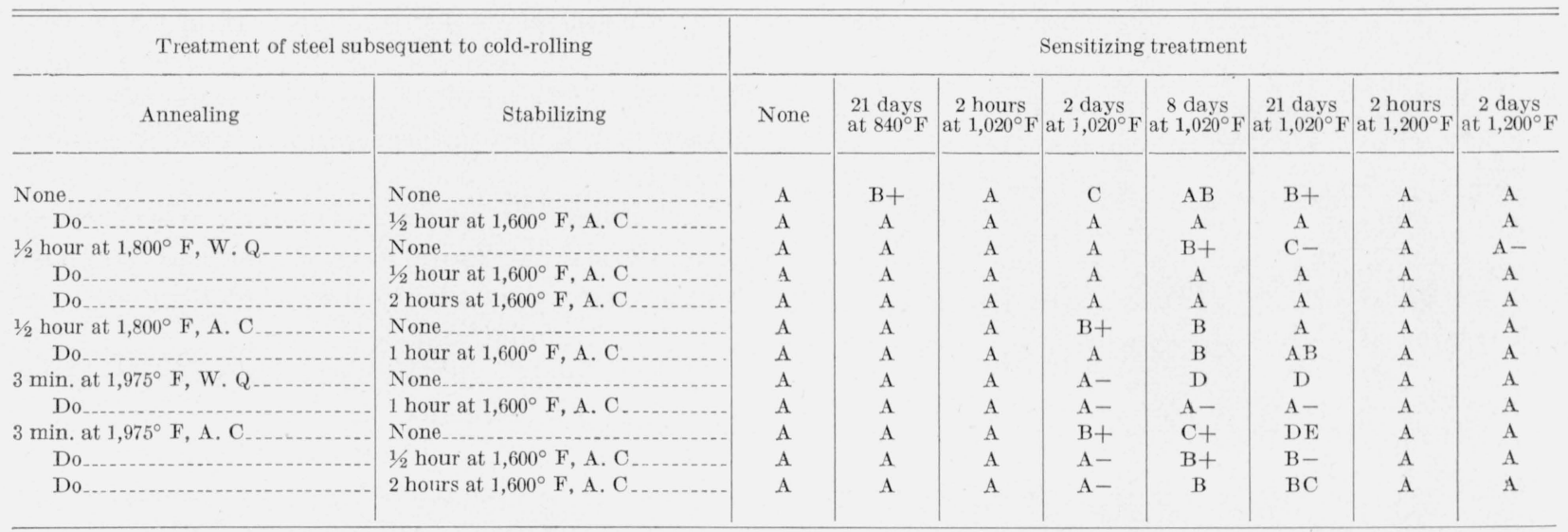

\section{Results and Discussion}

All specimens were examined for carbide distribution subsequent to sensitizing. The type of distribution of the precipitated carbides was not an infallible indication of the resistance to intergranular attack. Steels in which the carbides were distributed randomly were usually resistant to intergranular attack. However, steels that contained carbides at the grain boundaries, even though these carbides were distributed as a continuous network, either were or were not susceptible to intergranular attack, depending on the time-temperature relation during sensitization. Frequently the microstructures of susceptible and unsusceptible specimens of the same steel appeared quite similar. A rather striking illustration of this statement is shown in figure 2. This figure shows the microstructure of a straight carbon $(0.09 \%)$ austenitic steel, initially as cold rolled, after sensitizing 2 hours at $1,200^{\circ} \mathrm{F}$ (fig. 2, A) and after sensitizing 2 days at $1,200^{\circ} \mathrm{F}$ (fig. 2, B). Both micrographs show a precipitation of carbides at the grain boundaries and on various slip planes, and it is evident that the microstructures resulting from the two treatments are indistinguishable. The behavior of these two specimens after exposure to the boiling acidified copper sulfate solution, however, differed radically (fig. 2, c). The left side of the micrograph, which corresponds to specimen A, was exposed 2 days and suffered severe intergranular corrosion. The right side of the micrograph, which corresponds to specimen 

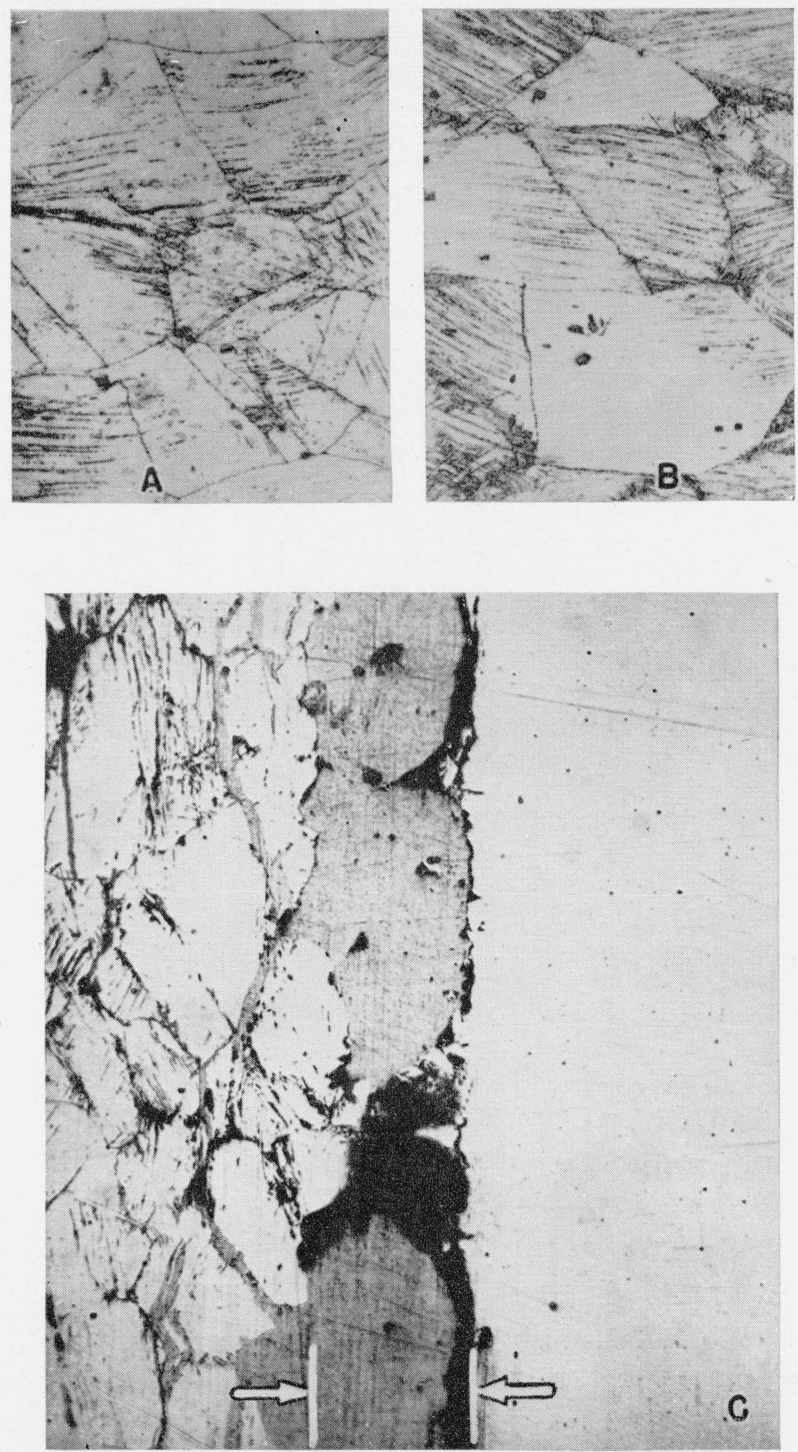

FIGURE 2. Structure of straight carbon (0.09\%) austenitic stainless steel, initially as cold-rolled, before and after exposure to the boiling acidified copper sulfate solution.

$A$, Sensitized 2 hours at $1,200^{\circ} \mathrm{F}$. Heavy carbide precipitation at grain boundaries and on slip planes. Etched electrolytically 10 minutes in 10-percent sodium cyanide. $\times 500$. $B$, Sensitized 2 days at $1,200^{\circ} \mathrm{F}$. Heavy carbide precipitation at grain boundaries and on slip planes. Etched electrolytically in 10-percent sodium cyanide. $\times 500$. $C$, specimens $A$ and $B$ after exposure to boiling acidified copper sulfate solution. Left side of micrograph, which corresponds to $A$, was exposed 2 days and suffered severe intergranular attack. Right side of micrograph, which corresponds to $B$, was exposed 14 days and showed no evidence of intergranular attack, yet both had the same type of carbide precipitation. The massive gray globules attached to specimen shown on left of $C$ are particles of copper deposited from the acidified copper sulfate solution. Unetched. $\times 250$.

B, was exposed 14 days and showed no evidence whatever of intergranular attack.

As a total of well over 2,500 individual specimens were tested, it is obviously impractical to present a detailed account of the test data procured. All data were assembled in tabular form for study. The performance of each specimen was then rated on the basis of each of the four methods of evaluation (electrical resistivity, metallic ring, bend test, and microstructure after corrosion). An arbitrary system of appraisal was used, as follows:

A. Completely immune to intergranular attack: Electrical resistivity, no increase; metallic ring, no impairment; bend test, no evidence of cracks; microstructure, no evidence of intergranular attack.

B. Practically immune to intergranular attack: Electrical resistivity, not more than 12 percent increase; metallic ring, slightly dead; bend test, slight cracks; microstructure, slight intergranular attack in extreme surface layers only.

C. Moderately vulnerable to intergranular attack: Electrical resistivity, more than 12 percent but not more than 25 percent increase; metallic ring, slight; bend test, cracked; microstructure, moderately severe intergranular attack in surface layers only.

D. Vulnerable to intergranular attack: electrical resistivity, more than 25 percent increase; metallic ring, dead; bend test, broke; microstructure, intergranular attack.

E. Extremely vulnerable; Same as D, but occurring in a few days.

The four ratings obtained for each individual specimen were then averaged by assigning numerical values to the ratings $(\mathrm{A}=4, \mathrm{~B}=3, \mathrm{C}=2$, $\mathrm{D}=1, \mathrm{E}=0$ ) and converting to the appropriate letter symbol. Plus or minus signs were used to indicate averages falling slightly above or below letters, respectively, and averages falling midway between two letters were denoted by both letters. These averages were then used to prepare tables 2 to 24 , inclusive, which summarize qualitatively the performance of each steel after various annealing, stabilizing, and sensitizing treatments. The averaged letter symbols may be considered as an order of merit, bearing in mind that the differences between $\mathrm{A}$ and $\mathrm{B}$ are rather slight, and those between D and E are very large. Since specimens were exposed to the boiling acidified copper-sulfate solution for a maximum time of 14 days, specimens that were rated $\mathrm{B}$ and $\mathrm{C}$, and in many cases even $\mathrm{D}$, might have been rated as satisfactory had they been subjected to the cor- 
roding solution for the more or less standardized time of only 2 days.

A noteworthy feature shown by these tables is that the sensitizing temperature of $1,200^{\circ} \mathrm{F}$, which is commonly specified, is too high to effect maximum susceptibility to intergranular attack. Exposure to a sensitizing temperature of $1,020^{\circ} \mathrm{F}$ is much more effective, provided the time of exposure is quite long. A period of 8 or 21 days at $1,020^{\circ} \mathrm{F}$ appeared to be the most severe sensitizing treatment of those used. It was not unusual for steels that showed complete immunity to intergranular attack after sensitizing either 2 hours or 2 days at $1,200^{\circ} \mathrm{F}$ to be definitely vulnerable to intergranular attack after sensitizing for either 8 or 21 days at $1,020^{\circ} \mathrm{F}$. It is possible that a shorter period of time at a temperature higher than $1,020^{\circ} \mathrm{F}$, but lower than $1,200^{\circ} \mathrm{F}$, would be equally effective in causing maximum sensitiza. tion. This possibility, however, was not explored during this investigation.

Considering the steels that contained no stabilizing elements (tables 2 to 6 , inclusive), it is apparent that all of these were quite vulnerable to intergranular attack after certain sensitizing treatments. Decrease in carbon content decreased the degree of vulnerability, but even the two very low-carbon steels tested were quite susceptible to attack. The ratio of chromium to nickel in steel C-1 (table 2) was such that, in the presence of low carbon, appreciable amounts of delta ferrite were present in the microstructure. Steel C-10 (table 3) contained lower chromium and higher nickel so that no delta ferrite existed. This latter steel proved generally to be the more susceptible to intergranular attack except that, in the initially cold-rolled condition, it was practically immune.

The performance of the individual columbiumand titanium-treated steels may be studied by reference to the appropriate table, but a more readily comprehensible presentation is given in table 25. In this table only the worst averaged rating of each steel, as presented in tables 7 to 24 , inclusive, is used. The steels are arranged in table 25 in order of $\mathrm{Cb} / \mathrm{C}$ or $\mathrm{Ti} / \mathrm{C}$ ratio without regard to the carbon content. It will be observed that, for all 12 initial conditions studied, the performance of the steels improves as the $\mathrm{Cb} / \mathrm{C}$ or $\mathrm{Ti} / \mathrm{C}$ ratios increase. Apparently the carbon content within the range studied (0.06 to $0.13 \%$ ) had little, if any, effect upon susceptibility to intergranular attack, the predominating factor being the $\mathrm{Cb} / \mathrm{C}$ or $\mathrm{Ti} / \mathrm{C}$ ratio. The ratings of the various steels, as given in table 25 , are plotted, as an order of merit, against the $\mathrm{Cb} / \mathrm{C}$ and $\mathrm{Ti} / \mathrm{C}$ ratios in figures 3 and 4 .

TABLE 25. Qualitative rating of resistance of test steels to intergranular attack a

\begin{tabular}{|c|c|c|c|c|c|c|c|c|c|c|c|c|c|c|c|}
\hline \multirow[b]{2}{*}{ Steel No. } & \multirow[b]{2}{*}{$\begin{array}{l}\text { Percent- } \\
\text { age of } C\end{array}$} & \multicolumn{2}{|c|}{ Ratio } & \multicolumn{2}{|c|}{$\begin{array}{l}\text { Cold-rolled } \\
(371 / 2 \%)\end{array}$} & \multicolumn{3}{|c|}{$\begin{array}{c}\text { Annealed } 1 / 2 \mathrm{hr} \text { at } 1,800^{\circ} \mathrm{F} \text {, } \\
\text { W. Q. }\end{array}$} & \multicolumn{2}{|c|}{$\begin{array}{l}\text { Annealed } 1 / 2 \mathrm{hr} \text { at } \\
1,800^{\circ} \mathrm{F} \text {, A. C. }\end{array}$} & \multicolumn{2}{|c|}{$\begin{array}{l}\text { Annealed } 3 \mathrm{~min} \text { at } \\
1,975^{\circ} \mathrm{F}, \mathrm{W} . \mathrm{Q} \text {. }\end{array}$} & \multicolumn{3}{|c|}{$\begin{array}{c}\text { Annealed } 3 \min \text { at } 1,975^{\circ} \mathrm{F} \text {, } \\
\text { A. C. }\end{array}$} \\
\hline & & $\mathrm{Cb} / \mathrm{C}$ & $\mathrm{Ti} / \mathrm{C}$ & $\begin{array}{l}\text { No sta- } \\
\text { bilizing } \\
\text { treat- } \\
\text { ment }\end{array}$ & $\begin{array}{l}\text { Stabil- } \\
\text { ized } 1 / 2 \\
\text { hr at } \\
1,600^{\circ} \mathrm{F} \text {. } \\
\text { A. C. }\end{array}$ & $\begin{array}{c}\text { No sta- } \\
\text { bilizing } \\
\text { treat- } \\
\text { ment }\end{array}$ & $\begin{array}{c}\text { Stabil- } \\
\text { ized } 1 / 2 \\
\text { hr at } \\
1,600^{\circ} \mathrm{F} \text {, } \\
\text { A. C. }\end{array}$ & $\begin{array}{c}\text { Stabil- } \\
\text { ized } 2 \\
\text { hr at } \\
1,600^{\circ} \mathrm{F} \\
\text { A. C. }\end{array}$ & $\begin{array}{c}\text { No sta- } \\
\text { bilizing } \\
\text { treat- } \\
\text { ment }\end{array}$ & $\begin{array}{c}\text { Stabil- } \\
\text { ized 1 } \\
\text { hr at } \\
1,600^{\circ} \mathrm{F}, \\
\text { A. C. }\end{array}$ & $\begin{array}{c}\text { No sta- } \\
\text { bilizing } \\
\text { treat- } \\
\text { ment }\end{array}$ & $\begin{array}{c}\text { Stabil- } \\
\text { ized 1 } \\
\text { hr at } \\
1,600^{\circ} \mathrm{F} \text {, } \\
\text { A. C. }\end{array}$ & $\begin{array}{c}\text { No sta- } \\
\text { bilizing } \\
\text { treat- } \\
\text { ment }\end{array}$ & $\begin{array}{c}\text { Stabil- } \\
\text { ized } 1 / 2 \\
\text { hr at } \\
1,600^{\circ} \mathrm{F} \\
\text { A. C. }\end{array}$ & $\begin{array}{c}\text { Stabil- } \\
\text { ized } 2 \\
\text { hr at } \\
1,600^{\circ} \mathrm{F}, \\
\text { A. C. }\end{array}$ \\
\hline $\mathrm{S}-26 \ldots$ & 0.068 & 7.4 & & $\mathrm{D}$ & $\mathrm{DE}$ & $\mathrm{E}$ & $\mathrm{E}+$ & $\mathrm{E}$ & E & $\mathrm{E}+$ & $\mathrm{E}$ & $\mathrm{DE}$ & $\mathrm{E}$ & $\mathrm{E}$ & $\mathrm{E}$ \\
\hline $\mathrm{S}-28 \ldots \ldots$ & .115 & 8.0 & & $\mathrm{C}-$ & $\mathrm{D}+$ & $\mathrm{E}$ & $\mathrm{E}+$ & $\mathrm{E}+$ & $\mathrm{E}+$ & $\mathrm{DE}$ & $\mathrm{E}$ & $\mathrm{E}$ & $\mathrm{E}$ & $\mathrm{E}+$ & $\mathrm{E}+$ \\
\hline S-6 $6 \ldots \ldots$ & .070 & 8.7 & & B & $\mathrm{C}-$ & $\mathrm{E}+$ & $\mathrm{E}+$ & $\mathrm{E}+$ & $\mathrm{D}-$ & $\mathrm{D}-$ & $\mathrm{E}$ & $\mathrm{E}$ & $\mathrm{E}+$ & $\mathrm{E}$ & $\mathrm{E}$ \\
\hline S $-27 \ldots \ldots$ & .087 & 8.7 & & B & $\mathrm{C}-$ & $\mathrm{E}+$ & $\mathrm{E}+$ & $\mathrm{E}+$ & & & - nen- & & & & \\
\hline S-17 ........ & .117 & 9.7 & & $\mathrm{~B}+$ & $\mathrm{A}$ & $\mathrm{B}+$ & $\mathrm{B}$ & A & $\mathrm{AB}$ & $\mathrm{BC}$ & $\mathrm{D}+$ & B & $\mathrm{CD}$ & $\mathrm{D}$ & $\mathrm{C}-$ \\
\hline S-12 ..... & .074 & 10.1 & & $\mathrm{~B}+$ & A & $A-$ & $A-$ & A & $A-$ & $\mathrm{B}-$ & $\mathrm{D}+1$ & B & $\mathrm{C}-$ & $\mathrm{C}-$ & $\mathrm{C}$ \\
\hline S-18 .... & .132 & 11.1 & & $A-$ & A & $A-$ & $A-$ & A & A & $\mathrm{B}+$ & B & $A-$ & $\mathrm{B}+$ & B & $\mathrm{A}-$ \\
\hline $\mathrm{C}-3 \ldots \ldots \ldots$ & .060 & 11.8 & & $A-$ & A & A & $A-$ & A & A & $A-$ & A & A & $A-$ & $\Lambda$ & A \\
\hline S-34 ... & .067 & & 3.9 & $\mathrm{E}+$ & $\mathrm{E}$ & $\mathrm{E}$ & $\mathrm{E}$ & $\mathrm{E}$ & $\mathrm{E}$ & $\mathrm{E}$ & $\mathrm{E}$ & $\mathrm{E}$ & $\mathrm{E}$ & $\mathrm{E}$ & $\mathrm{E}$ \\
\hline S- $-35 \ldots$. & .107 & & 4.1 & $\mathrm{E}+$ & $\mathrm{E}$ & $\mathrm{E}$ & $\mathrm{E}$ & $\mathrm{E}$ & $\mathrm{E}$ & $\mathrm{DE}$ & $\mathrm{E}$ & $\mathrm{E}$ & $\mathrm{E}$ & $\mathrm{E}$ & $\mathrm{E}$ \\
\hline S-21 $\ldots \ldots$ & .071 & $\ldots$. & 4.5 & $\mathrm{DE}$ & $\mathrm{E}+$ & $\mathrm{E}$ & . $\mathrm{E}$ & $\mathrm{E}$ & $\mathrm{E}$ & $\mathrm{D}-$ & $\mathrm{E}$ & $\mathrm{E}$ & $\mathrm{E}+$ & $\mathrm{E}$ & $\mathrm{E}$ \\
\hline $\mathrm{S}-25 \ldots \ldots$ & .082 & -.... & 4.5 & $\mathrm{D}+$ & $\mathrm{E}+$ & $\mathrm{E}$ & $\mathrm{E}$ & $\mathrm{E}+$ & & & & & & & \\
\hline $\mathrm{S}-36 \ldots \ldots \ldots$ & .109 & $\ldots$ & 5. 0 & $\mathrm{D}-$ & $\mathrm{A}$ & $\mathrm{E}+$ & $\mathrm{A}$ & A & $\mathrm{C}+$ & B & $\mathrm{E}$ & $\mathrm{B}$ & $\mathrm{E}$ & $\mathrm{BC}$ & $\mathrm{C}-$ \\
\hline S-39 . . . & .065 & -.... & 5.5 & $\mathrm{~B}+$ & A & $\mathrm{C}-$ & $\mathrm{BC}$ & $\mathrm{BC}$ & $\mathrm{CD}$ & $\mathrm{BC}$ & $\mathrm{E}$ & $\mathrm{C}$ & $\mathrm{E}$ & $\mathrm{D}$ & $\mathrm{CD}$ \\
\hline $\mathrm{C}-6 \ldots \ldots \ldots$ & .064 & -.... & 5.5 & $A-$ & A & $\mathrm{C}-$ & $A-$ & $\mathrm{A}-$ & $\mathrm{C}-$ & $\mathrm{BC}$ & $\mathrm{D}-$ & $\mathrm{C}-$ & $\mathrm{E}+$ & $\mathrm{D}$ & $\mathrm{C}+$ \\
\hline $\mathrm{S}-32 \ldots \ldots \ldots$ & .105 & -.... & 5.8 & $\mathrm{C}$ & $\mathrm{A}$ & $\mathrm{C}-$ & A & A & B & B & $\mathrm{D}$ & $A-$ & DE & $\mathrm{B}-$ & $\mathrm{BC}$ \\
\hline $\mathrm{C}-2 \ldots \ldots \ldots$ & .070 & -..... & 7.1 & $\mathrm{C}-$ & $\mathrm{A}$ & A & A & A & $\mathrm{B}+$ & $A-$ & $\mathrm{D}$ & $A-$ & $\mathrm{D}+$ & $A-$ & $A-$ \\
\hline $\mathrm{C}-8 \ldots \ldots \ldots$ & .075 & $|\ldots|$ & 7.9 & $\mathrm{~B}+$ & A & A & $A-$ & A & $\mathrm{A}-$ & $\mathrm{A}-$ & $A-$ & A & B & A & $\mathrm{A}-$ \\
\hline
\end{tabular}

a This table was prepared from tables 7 to 24 , inclusive, using only the worst averaged rating of each steel in each initial condition. For instance, steel S-26, as cold-rolled and stabilized $1 / 2$ hour at $1,600^{\circ} \mathrm{F}$, was rated DE as tested after sensitizing 8 days at $1,020^{\circ} \mathrm{F}$ (see table 7 ). Although for all other conditions of sensitization the ratings were higher than DE, this worst rating was used in this summary table. 


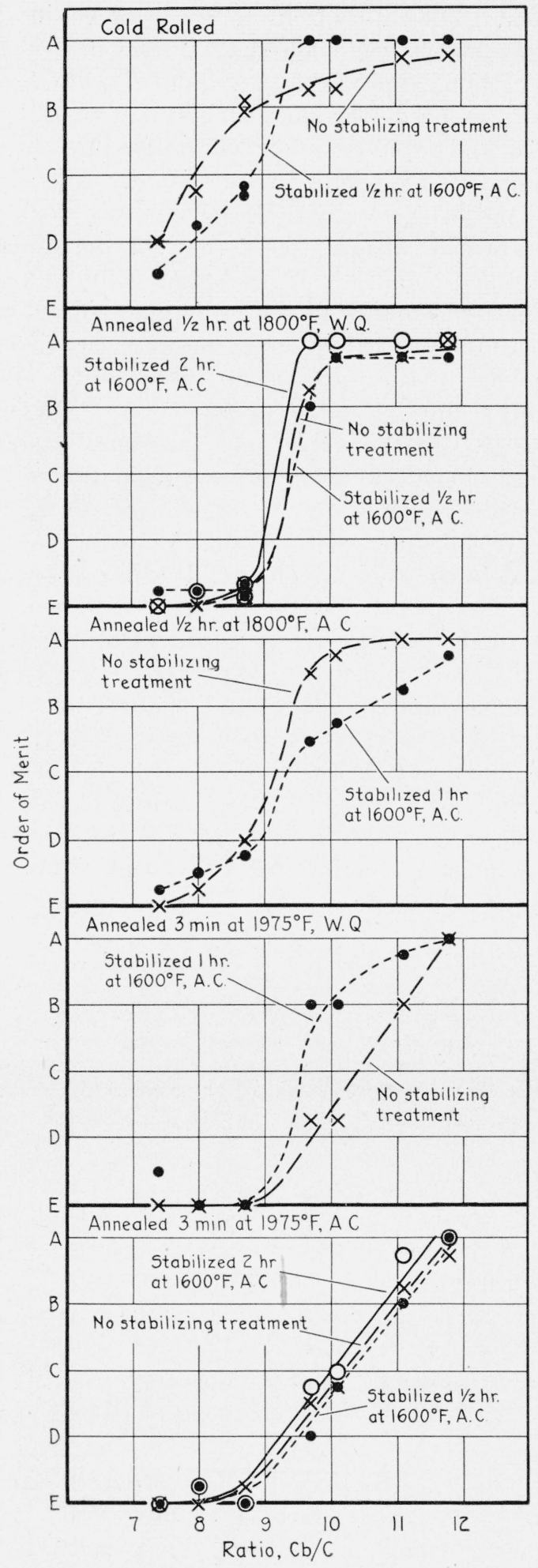

FIGURE 3. Influence of ratio of $\mathrm{Cb} / \mathrm{C}$ and of initial heat treatment upon resistance to intergranular attack.

See page 334 for explanation of significance of letters representing order of merit.

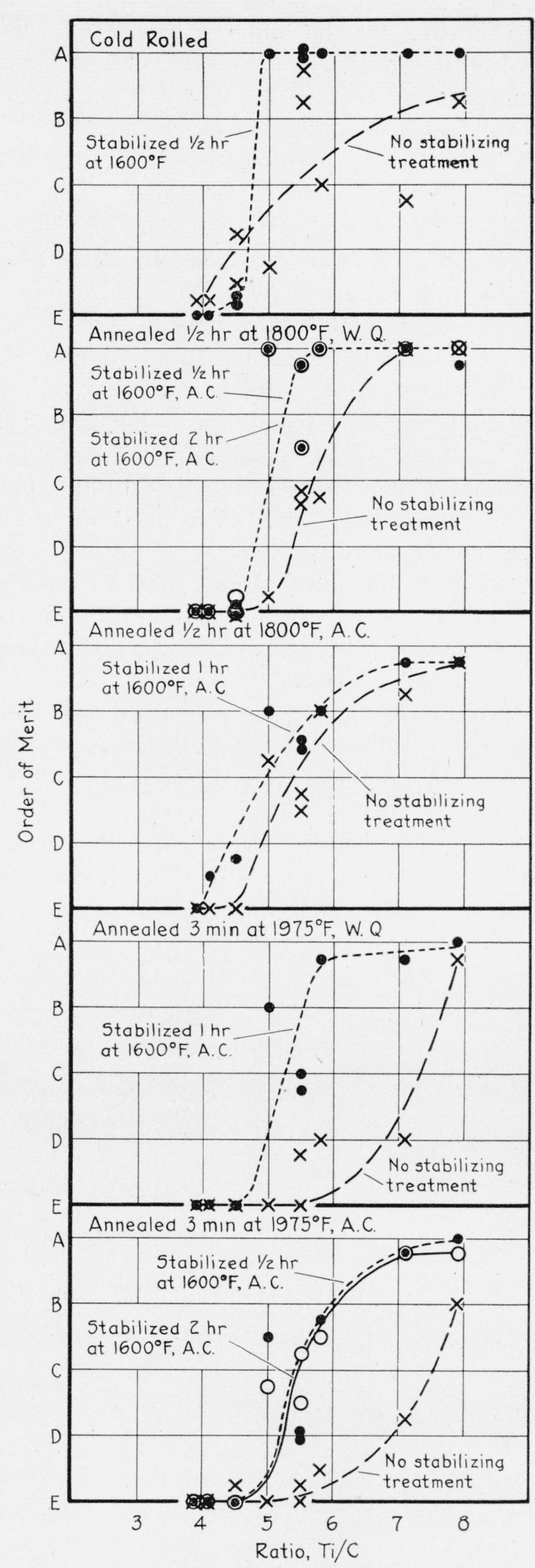

FIgure 4. Influence of ratio of $\mathrm{Ti} / \mathrm{C}$ and of initial heat treatment upon resistance to intergranular attack.

See page 334 for explanation of significance of letters representing order of merit. 
Regardless of the initial condition of the steels, increase in $\mathrm{Cb} / \mathrm{C}$ or $\mathrm{Ti} / \mathrm{C}$ to the higher ratios had a markedly beneficial effect upon the resistance to intergranular embrittlement. The ratio required for substantial immunity varied with the initial condition of the steel. Of the initial conditions studied, those conferring maximum immunity to intergranular attack were as cold rolled or as quenched from $1,800^{\circ} \mathrm{F}$ in water. Increase in annealing temperature from $1,800^{\circ}$ to $1,975^{\circ} \mathrm{F}$ impaired the resistance to intergranular attack. The deleterious effect of higher annealing temperatures upon susceptibility to intergranular attack has been shown by other investigators [8]. Air cooling instead of water quenching after annealing: was also detrimental. The stabilizing treatment appeared to be quite necessary for the titaniumtreated steels but was generally of only slight benefit to the columbium-treated steels. In fact, a superimposed stabilizing treatment appeared actually to be detrimental to the columbiumtreated steels as air cooled from $1,800^{\circ} \mathrm{F}$. The data indicated no significant differences between stabilizing periods of $1 / 2$ and 2 hours at $1,600^{\circ} \mathrm{F}$.

\section{Summary and Conclusions}

Data are presented on the resistance to intergranular embrittlement of 23 austenitic corrosionresisting steels $(18 \% \mathrm{Cr}-10 \%$ Nibase composition) in 12 different initial conditions. Susceptibility to intergranular attack was determined after seven different sensitizing treatments, followed by a maximum of 14 days exposure in a boiling acidified copper sulfate solution. The following conclusions appear warranted:

1. Of the various sensitizing treatments utilized, maximum susceptibility to intergranular attack was developed by either 8 or 21 days at $1,020^{\circ} \mathrm{F}$. The commonly used sensitizing treatment of 2 hours at $1,200^{\circ} \mathrm{F}$ was ineffective in developing susceptibility in any but the most vulnerable steels.

2. The straight carbon austenitic corrosionresisting steels were very susceptible to intergranular embrittlement. The susceptibility decreased as the carbon content decreased but, with the exception of one low-carbon steel in the coldrolled condition only, even the very low carbon steels $(0.025 \%$ C) were vulnerable regardless of heat treatment.
3. In the columbium- and titanium-bearing steels, carbon content within the range of 0.06 to 0.13 percent had no influence upon the resistance to intergranular attack, except insofar as it influenced the $\mathrm{Cb} / \mathrm{C}$ or $\mathrm{Ti} / \mathrm{C}$ ratios. Steels having similar ratios of stabilizing element to carbon had approximately the same degree of susceptibility to intergranular attack regardless of the carbon content.

4. Both the columbium- and titanium-treated steels exhibited greater resistance to intergranular attack as cold rolled or annealed at $1,800^{\circ} \mathrm{F}$ and water quenched than as annealed at $1,800^{\circ} \mathrm{F}$ and air cooled, or as annealed at $1,975^{\circ} \mathrm{F}$ and either water quenched or air cooled.

5. Stabilizing heat treatments at $1,600^{\circ} \mathrm{F}$ had a negligible effect upon the resistance to intergranular embrittlement of the columbium-treated steels. The performance of the titanium-treated steels carrying the higher ratios of $\mathrm{Ti} / \mathrm{C}$, however, was markedly improved by such treatments. Variation in time of the stabilizing treatments from $1 / 2$ to 2 hours had no effect.

6 . When properly treated, substantially complete immunity to intergranular attack may be obtained with a minimum ratio of $\mathrm{Cb} / \mathrm{C}=10$ and $\mathrm{Ti} / \mathrm{C}=5$. For more "fool-proof" immunity, it is believed that these ratios should be 12 and 8 respectively.

It should be emphasized that the data and conclusions given in this paper refer only to the susceptibility of the test steels to intergranular attack as developed under test conditions considerably more severe than those normally used.

The authors are indebted to Lt. Dennis J. Carney and James Darby, Naval Research Laboratory, for assistance in melting the experimental steels; to G. N. Goller, Rustless Iron and Steel Division, American Rolling Mill Co., Baltimore, Md., for forging many of the steels; to M. E. Carruthers and H. I. White, American Rolling Mill Research Laboratory, Middletown, Ohio, for assistance in rolling the experimental steels; to J. L. Hague and J. T. Sterling, National Bureau of Standards, for the chemical and vacuum fusion analyses, respectively, and to Albert Lewis, National Bureau of Standards, for assistance in the early phases of the work. 
This investigation was made possible by the generous support of the Bureau of Aeronautics, Navy Department, and the friendly cooperation of N. E. Promisel and B. A. Kornhauser of that Bureau.

\section{References}

[1] B. Strauss, H. Schottky, and J. Hinnüber, Z. anorg. Chem. 188, 309 (1930).

[2] Robert H. Aborn and Edgar C. Bain, Trans. Am. Soc. Steel Treating 18, 837 (1930).

[3] E. Houdremont and P. Schafmeister, Arch. Eisenhüttenswsen I, 187 (1933).
[4] H. D. Newell, Trans. Am. Soc. Steel Treating 19, 673 (1932).

[5] P. Payson, Trans. Am. Inst. Mining Met. Engineers 100, 306 (1932).

[6] E. C. Bain, R. H. Aborn, and J. J. B. Rutherford, Trans. Am. Soc. Steel Treating 21, 481 (1933).

[7] Frederick M. Becket and Russell Franks, Trans. Am. Inst. Mining Met. Engineers 113, 143 (1934).

[8] L. B. Pfeil and D. G. Jones, J. Iron Steel Inst. 127, 337 (1933).

Washington, December 16, 1947. 